\title{
CLIMATIC DATA FOR WILLIAMS LAKE, HUBBARD COUNTY, MINNESOTA, 1987-88
}

By R.S. Parkhurst, D.A. Merk, D.O. Rosenberry, and T.C. Winter U.S. GEOLOGICAL SURVEY Open-File Report 92-475

Lakewood, Colorado 1992 


\section{DEPARTMENT OF THE INTERIOR \\ MANUEL LUJAN, JR., Secretary \\ U.S. GEOLOGICAL SURVEY \\ Dallas L. Peck, Director}

For additional information write to:

Thomas C. Winter

U.S. Geological Surevey, MS 413

Box 25046, Denver Federal Center

Denver, CO 80025
For sale by:

Open-File Services Section

Western Distribution Branch

U.S. Geolocal Survey, MS 306

Box 25425, Federal Center

Denver, CO 80225 


\section{CONTENTS}

Page

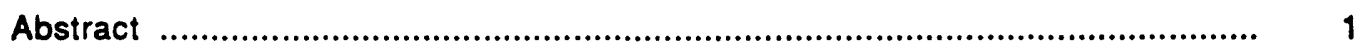

Introduction

Data collection and presentation ............................................................... $\quad 2$

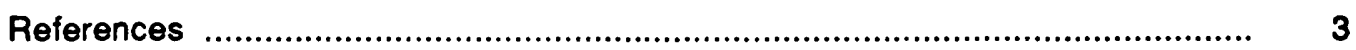

\section{ILLUSTRATIONS}

Figure 1. Map showing locations of Williams Lake and National Weather

Service precipitation sites, and instrument stations

\section{TABLES}

Table 1. Air temperature, vapor pressure, and humidity from the raft station at Williams Lake, 1987-88

2. Wind speeds at raft station on Williams Lake, 1987-88

3. Precipitation at Williams Lake gage and nearby National Weather

Services gages ,1987-88

\section{CONVERSION FACTORS}

$\begin{array}{lll}\text { Multiply } & \text { By } & \text { To Obtain } \\ \text { meter } & 3.281 & \text { feet } \\ \text { kilopascal } & 0.0099 & \text { standard atmosphere } \\ \text { mile per hour } & 1.609 & \text { kilometer per hour }\end{array}$

To convert degrees Celsius $\left({ }^{\circ} \mathrm{C}\right)$ to degress Fahrenheit $\left({ }^{\circ} \mathrm{F}\right)$ use the followind formula:

$$
{ }^{\circ} \mathrm{F}=9 / 5\left({ }^{\circ} \mathrm{C}\right)+32
$$




\section{CLIMATIC DATA FOR WILLIAMS LAKE, HUBBARD COUNTY, MINNESOTA \\ 1987-88}

by R.S. Parkhurst, D.A. Merk, D.O. Rosenberry, and T.C. Winter

ABSTRACT

Research on the hydrology of Williams Lake, north-central Minnesota, includes the study of evaporation. Presented here are those climatic data needed for mass-transfer studies, including: air temperatures, wind speed, humidity, and precipitation. Some calculated values necessary for this study, such as vapor pressure and vapor pressure deficit, are presented also. Data were collected at raft and land stations.

\section{INTRODUCTION}

Climatic data are being collected at Williams Lake, Hubbard County, Minnesota (fig.1), as part of a continuing study by the U.S. Geological Survey of the hydrology of the lake. Williams Lake is one of several lakes in different parts of the United States that have been selected for intensive study of hydrological, and related chemical and biological processes. The rationale for selection of Williams Lake is given by Siegel and Winter (1980). Climatic data from the 1982 and 1986 season are presented in Rosenberry and others (1988a, 1988b). Data collected in 1983, 1984, and 1985 are presented in Sturrock and others $(1984,1986 \mathrm{a}$, and 1986b). Because data from these reports were used for energy-budget studies, they contain additional data, such as long and short wave radiation, temperature of the lake surface, dry-bulb and wet-bulb temperature, and Bowen ratio numbers. Climatic data collected in 1987 and 1988 were used for mass-transfer studies.

\section{DATA COLLECTION AND PRESENTATION}

Data presented here are being collected principally for studies of evaporation. Therefore, the period of record includes only the time when the lake is ice-free. The period of record for 1987 is from April 28 (Julian day 118) to October 31 (Julian day 304). The period of record for 1988 is from April 25 (Julian day 116) to November 
(Julian day 312). Precipitation data from National Weather Service sites (fig. 1) are used in conjunction with precipitation data collected at the site and are collected throughout the entire year.

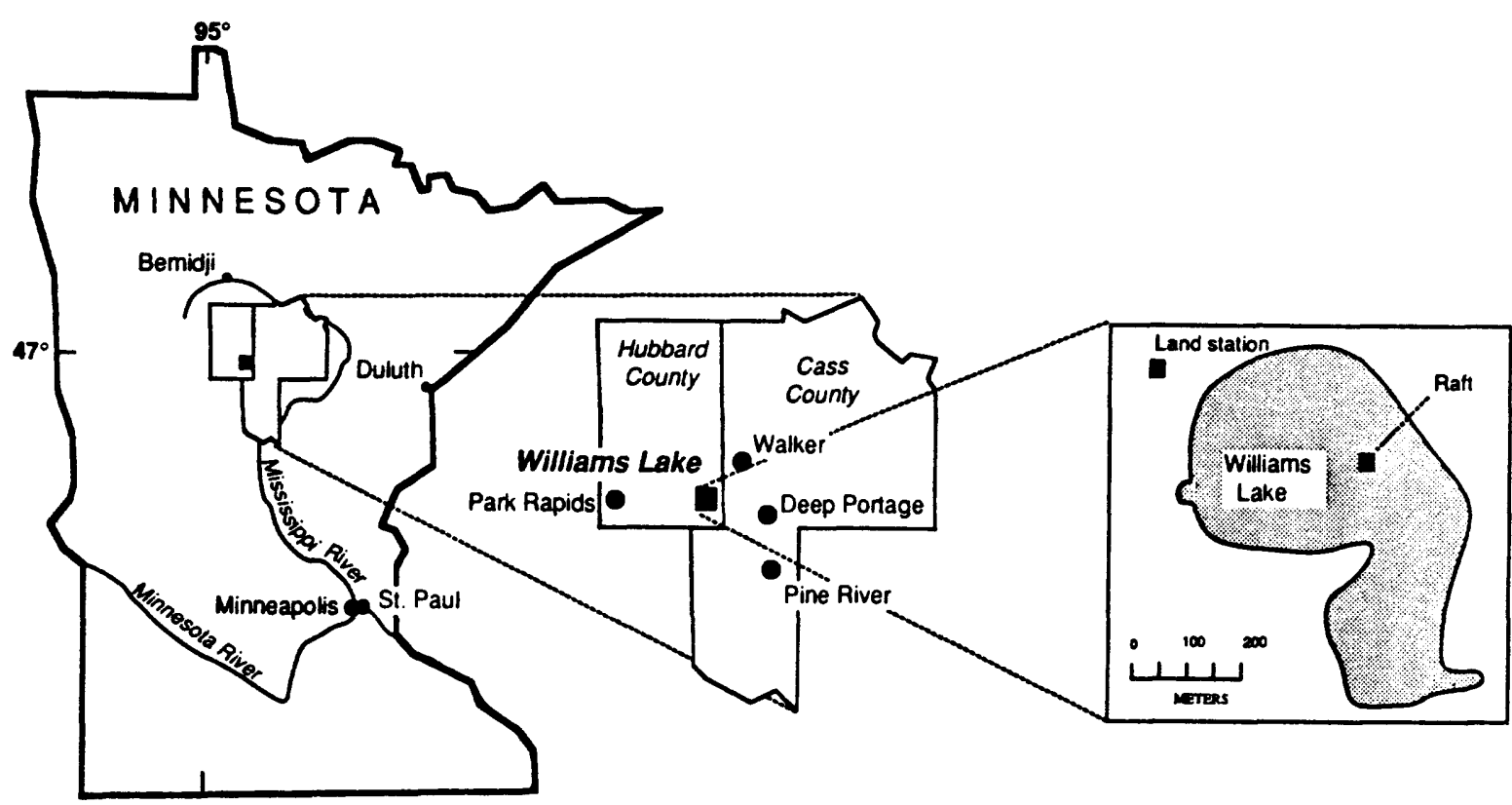

Figure 1. Locations of Williams Lake, National Weather Service precipitation sites, and instrument stations.

Climatological instruments are located on a raft in the middle of the lake, and at a land station located near the northwest shore of the lake (fig. 1). Instruments on the raft include anemometers at 1 and 2 meters above the water surface and an air temperature and humidity probe located 2 meters above the lake surface. Data from these sensors are recorded by a digital data logger located on the raft. The data logger scans the sensors every minute and calculates hourly and daily averages. In addition, for selected sensors, the daily output includes maximum and minimum values and the minute they occur. 
The land station, located in a field 135 meters northwest of the lake, consists of a tipping-bucket rain gage and an analog hygrothermograph. The tipping-bucket rain gage is connected to an event recorder The analog hygrothermograph measures air temperature and humidity, which are used as backup data. Both the event recorder and the hygrothermograph record the data on paper charts. Calibration checks are made with independent laboratory thermometers and an Assman psychrometer every few days.

Data presented here are daily summaries. Raft-station data together with landstation precipitation are considered primary. When the primary instruments were not operating properly, daily values were obtained by regression using data from backup instruments, if a satisfactory statistical relation could be established. Data used to establish regressions were selected so that they bracketed the period of missing or inadequate data. The values obtained by regression are noted in the tables.

Although only daily values are reported here, hourly values also were recorded. Hourly values are available for all or part of the period of record on request.

\section{REFERENCES}

Rosenberry, D.O., Sturrock, A.M., Scarborough, J.L., and Winter, T.C., 1988a, Climatic data for Williams Lake, Hubbard County, Minnesota, 1982: U.S. Geological Survey Open-File Report 88-89, 43 p.

Rosenberry, D.O., Sturrock, A.M., and Winter, T.C., 1988b, Climatic data for Williams Lake, Hubbard County, Minnesota, 1986: U.S. Geological Survey Open-File Report 88-304, $38 \mathrm{p}$.

Siegel, D.I., and Winter T.C., 1980, Hydrologic setting of Williams Lake, Hubbard County, Minnesota: U.S. Geological Survey Open-File Report 80-403, 56 p.

Sturrock, A.M., Rosenberry, D.O., Engelebrecht, L.G., Gothard, W.A., and Winter, T.C., 1984, Climatic data for Williams Lake, Hubbard County, Minnesota, 1983: U.S. Geological Survey Open-File Report 84-247, 41 p. 
Sturrock, A.M., Rosenberry, D.O., Scarborough, J.L., and Winter, T.C., 1986a, Climatic data for Williams Lake, Hubbard County, Minnesota, 1984: U.S. Geological Survey Open-File Report 86-64, 63 p.

Sturrock, A.M., Rosenberry, D.O., and Winter, T.C., 1986b, Climatic data for Williams Lake, Hubbard County, Minnesota, 1985: U.S. Geological Survey Open-File Report 86-607, $40 \mathrm{p}$. 


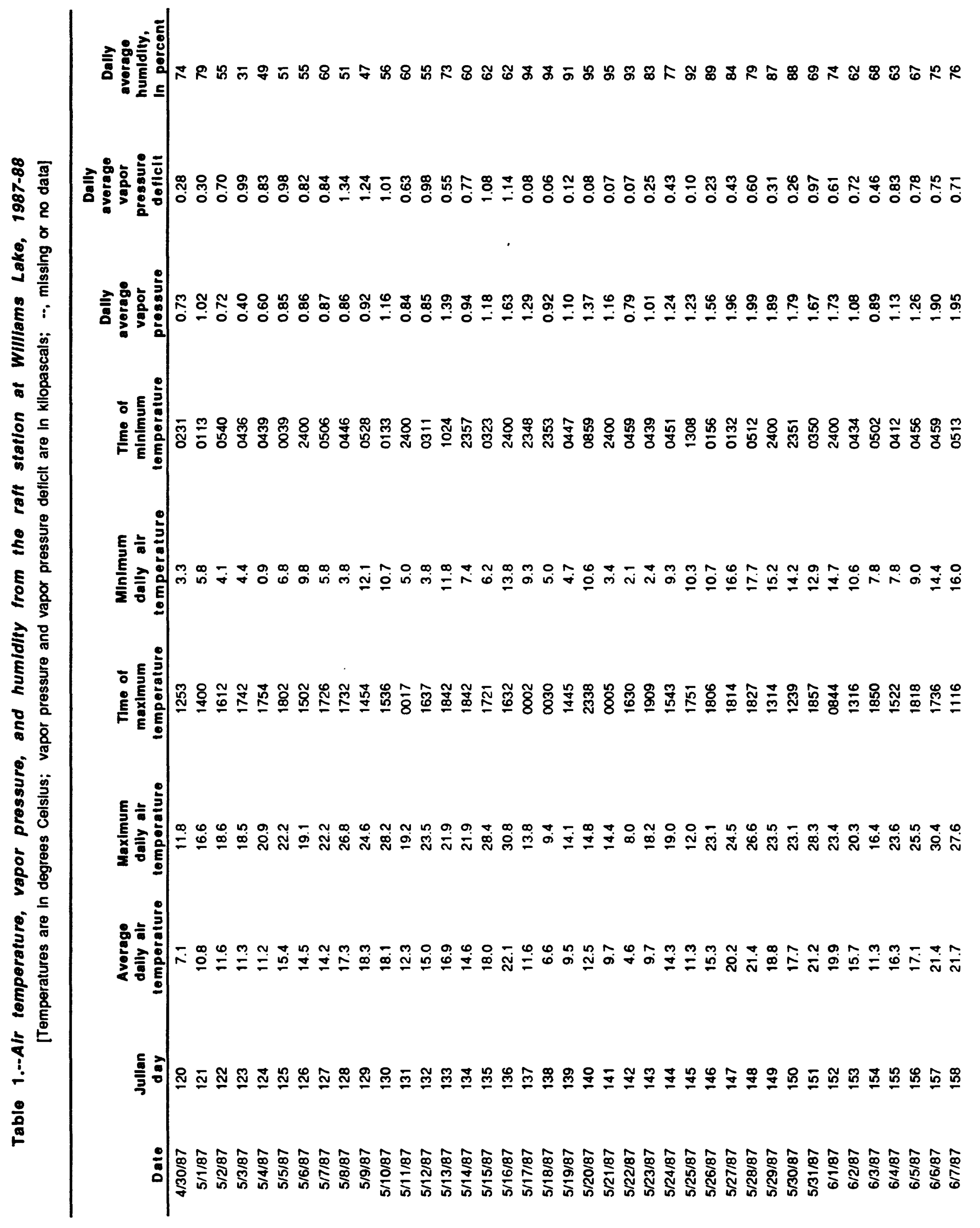




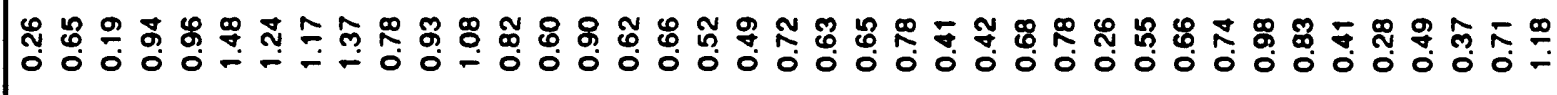

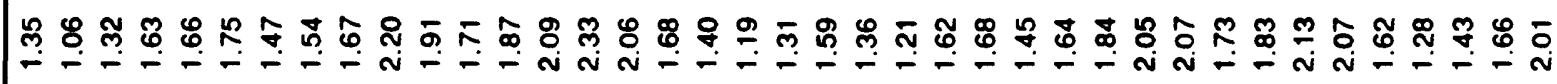

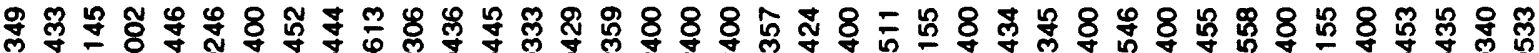

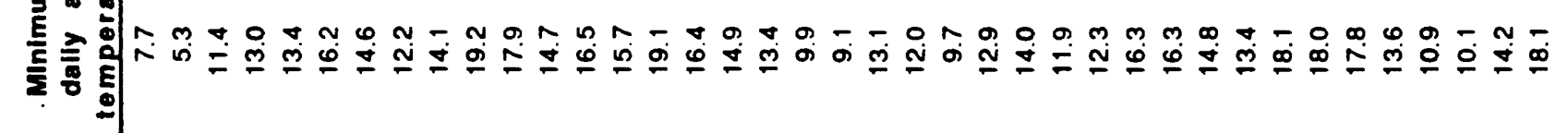

$\underline{\underline{2}}$

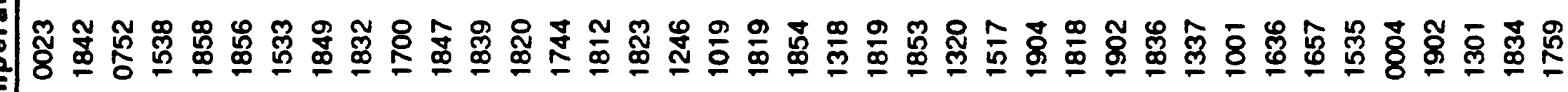

ำ

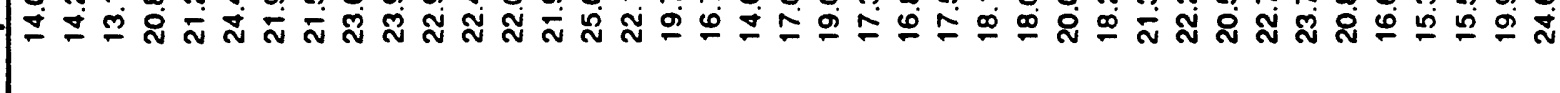




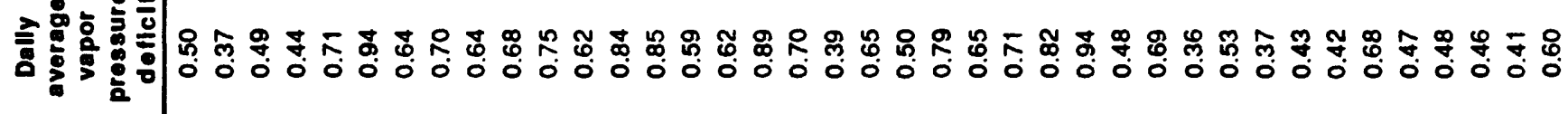

ㅇํำㅇำ

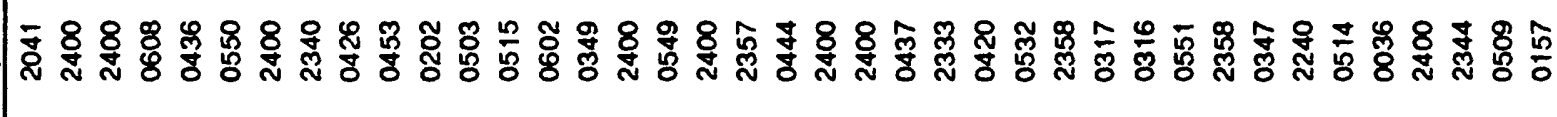




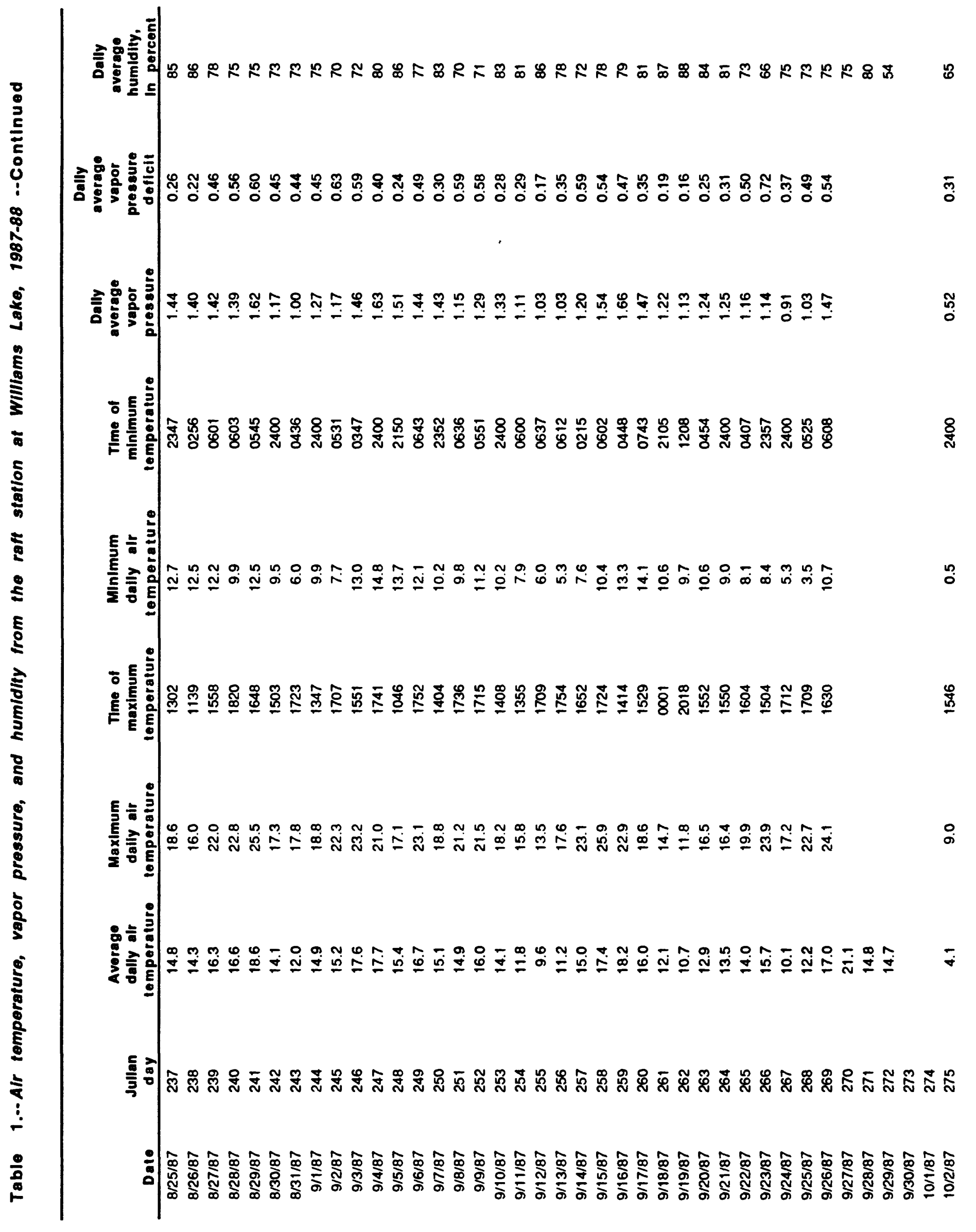




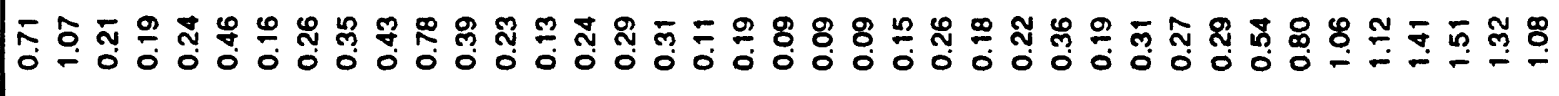

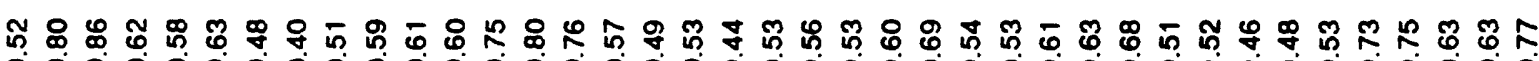

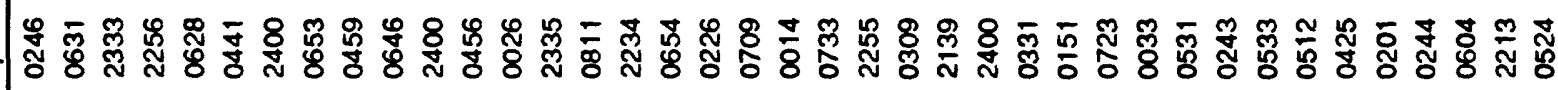

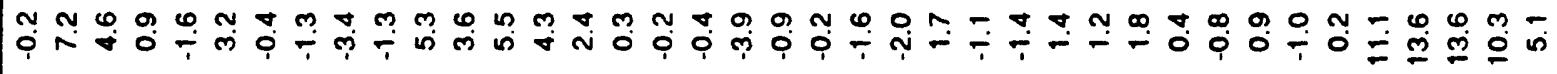

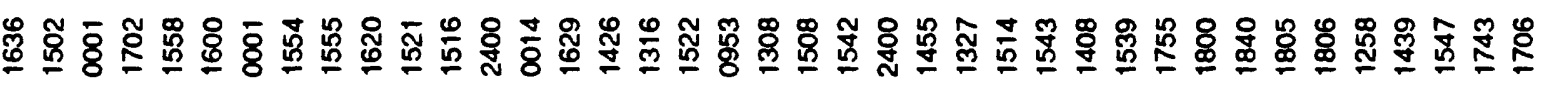

m $0-\sim 0$ -

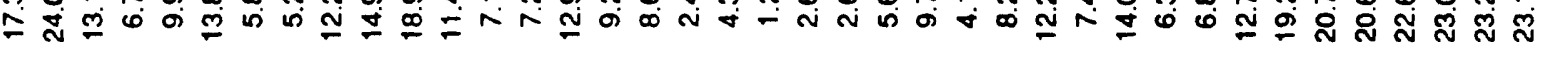

国

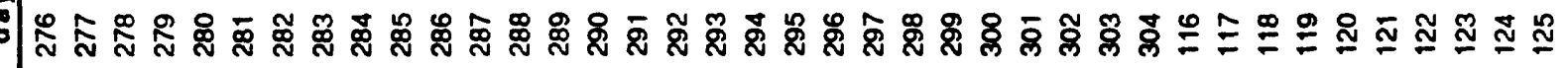

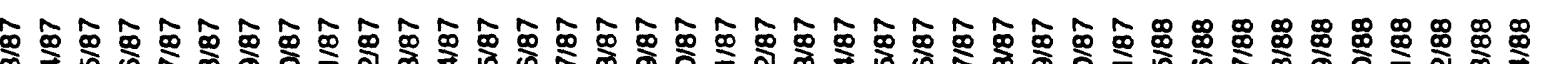

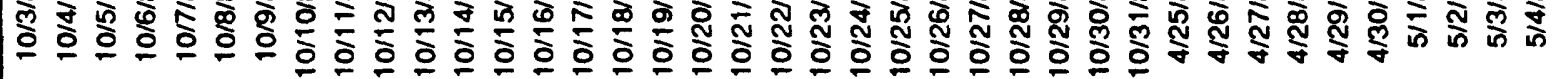




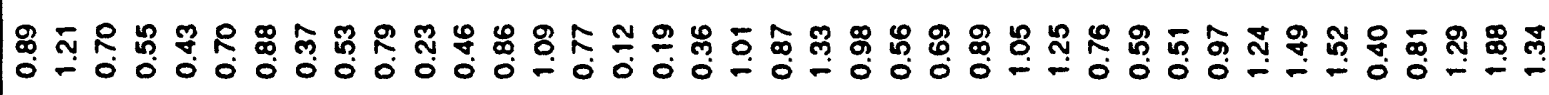

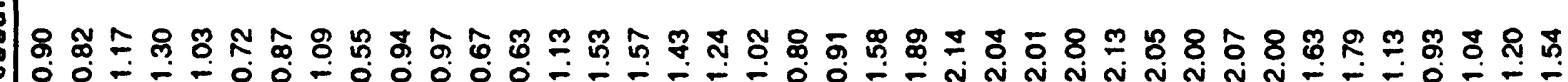

$0=-$

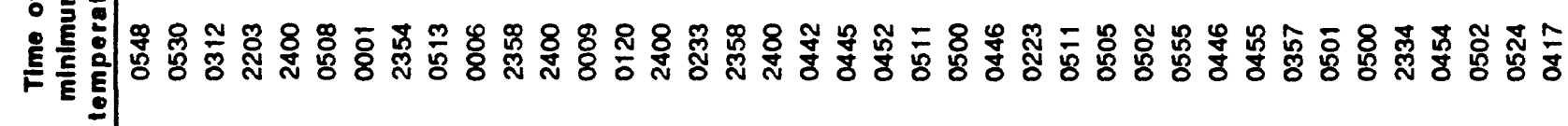

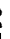

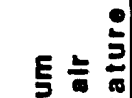

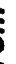

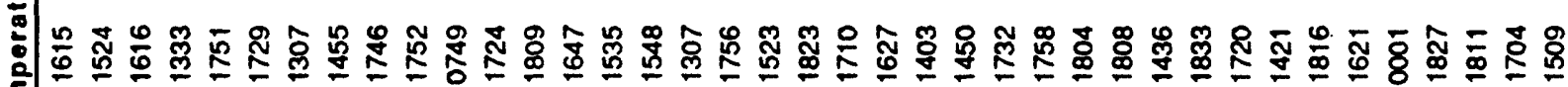




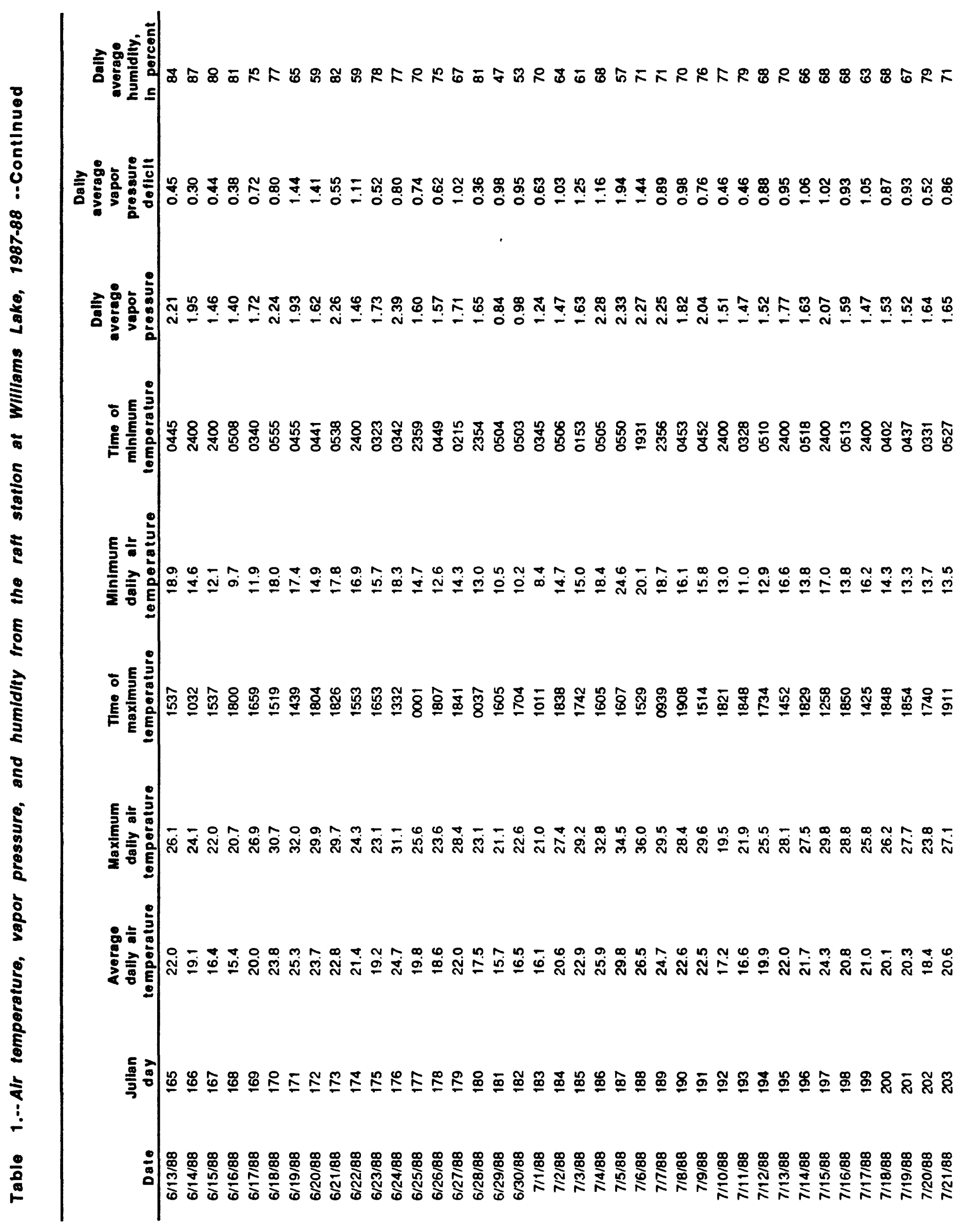




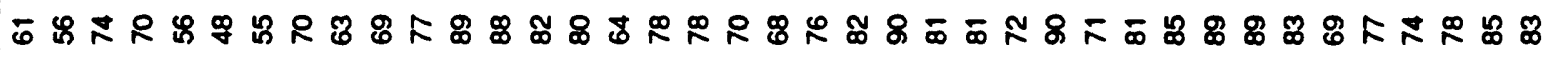

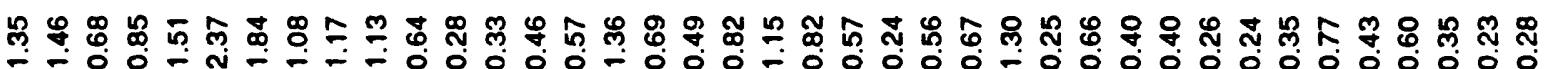

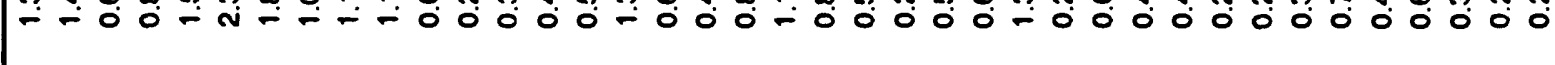

윰 ํㅜㄴ ํㅜㅇ

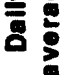

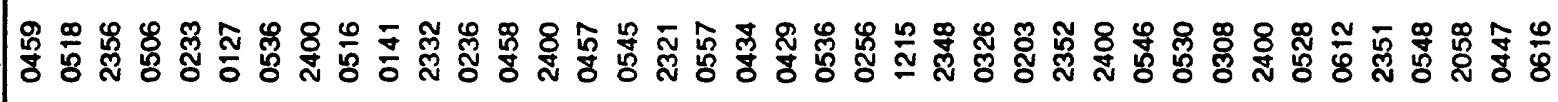

D.

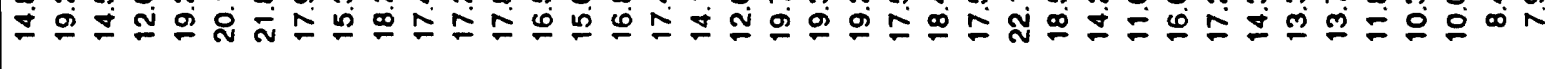

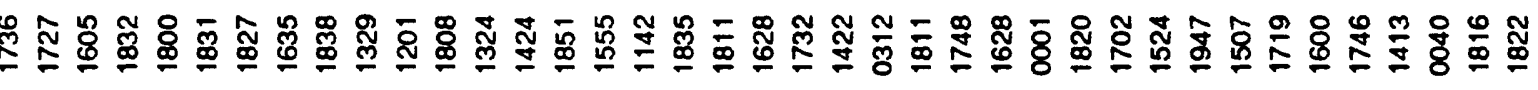

ก

ล ล

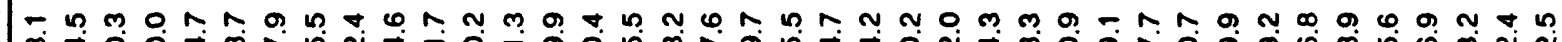

ฟ

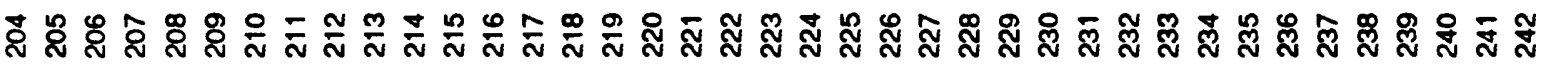

吕

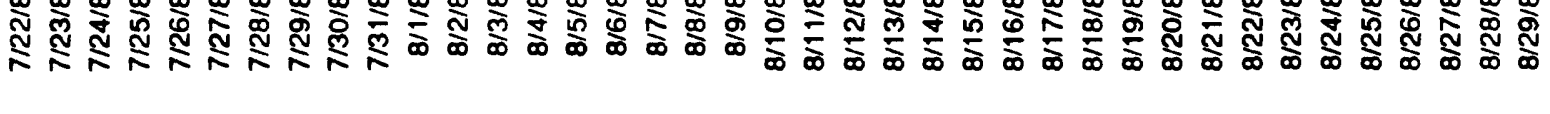




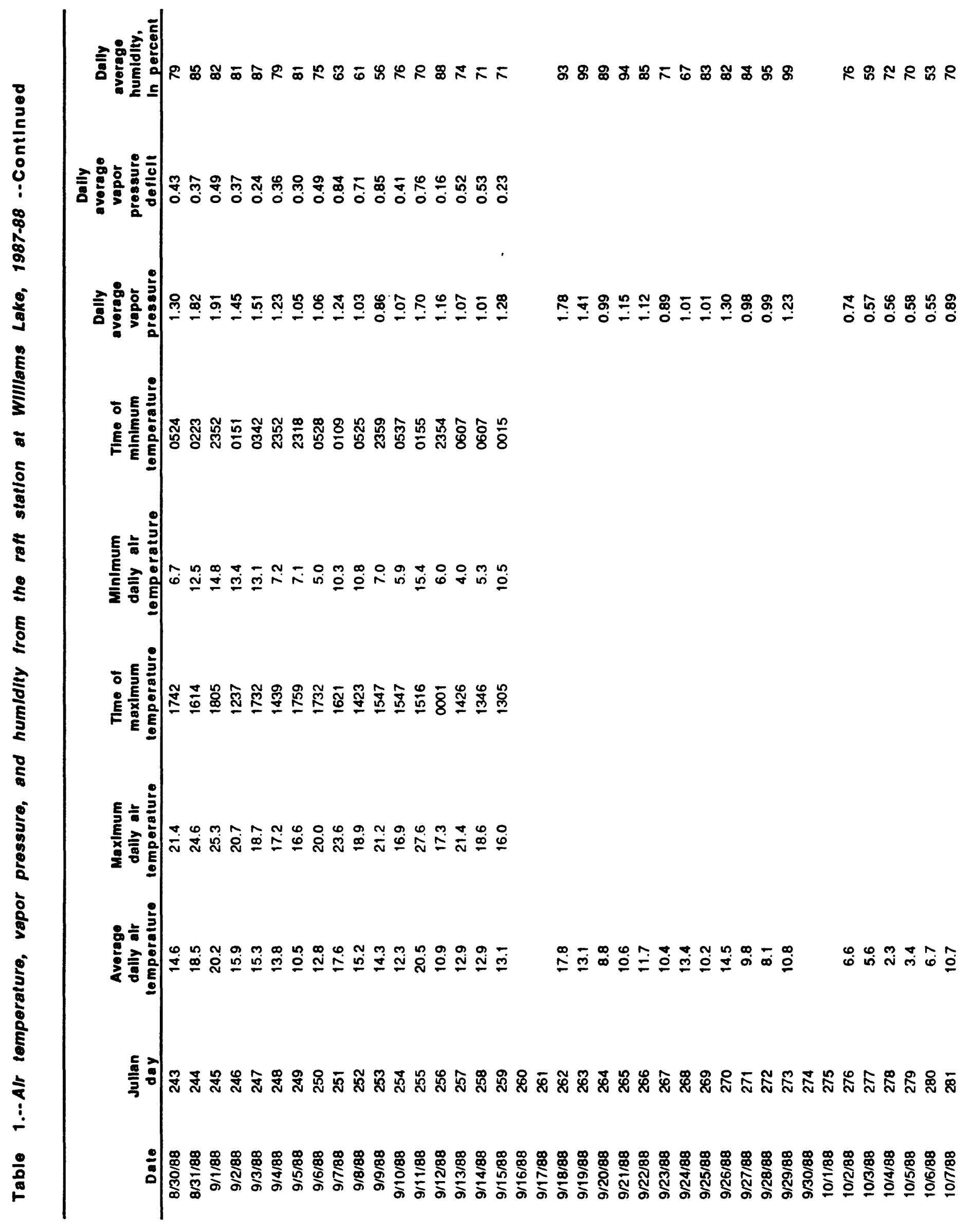




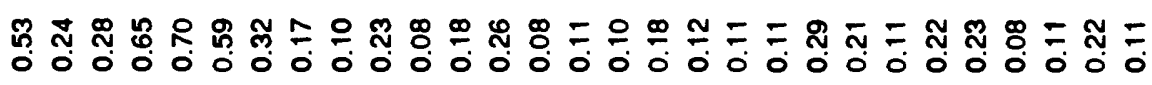

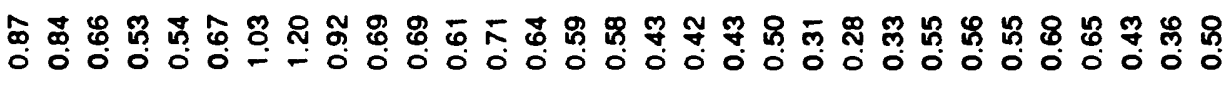

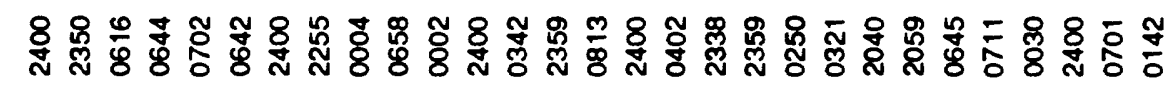

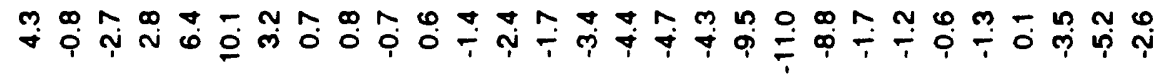

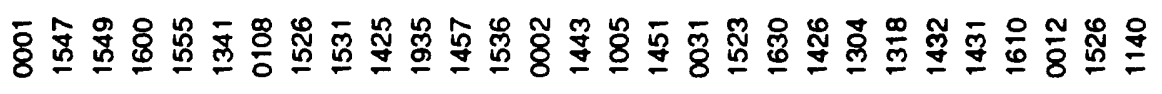

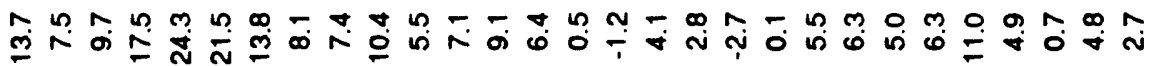

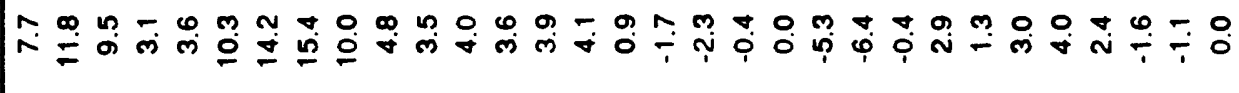

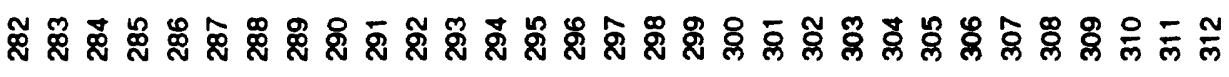

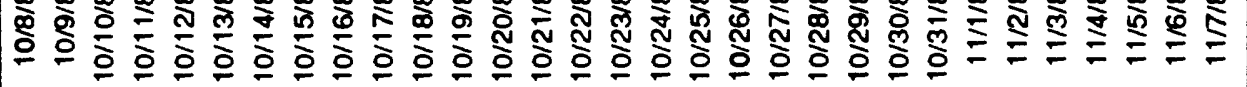




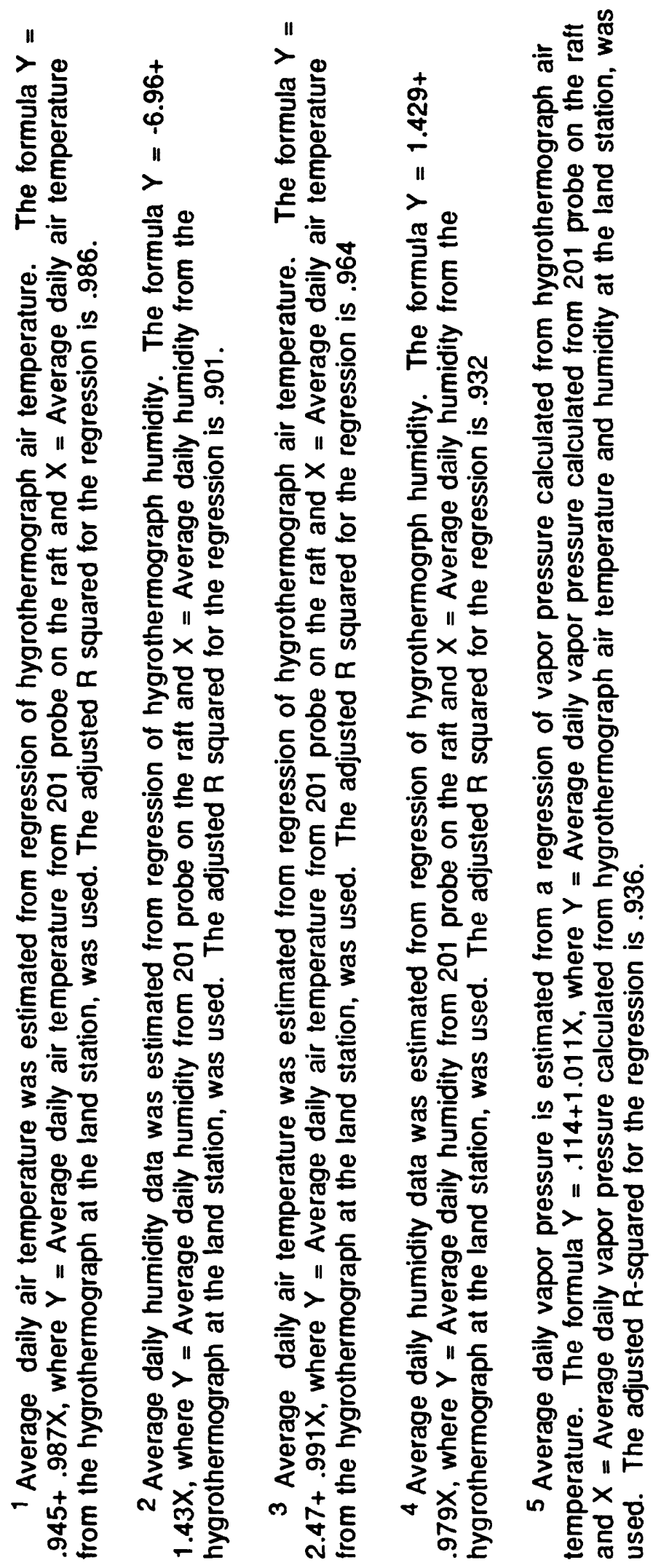




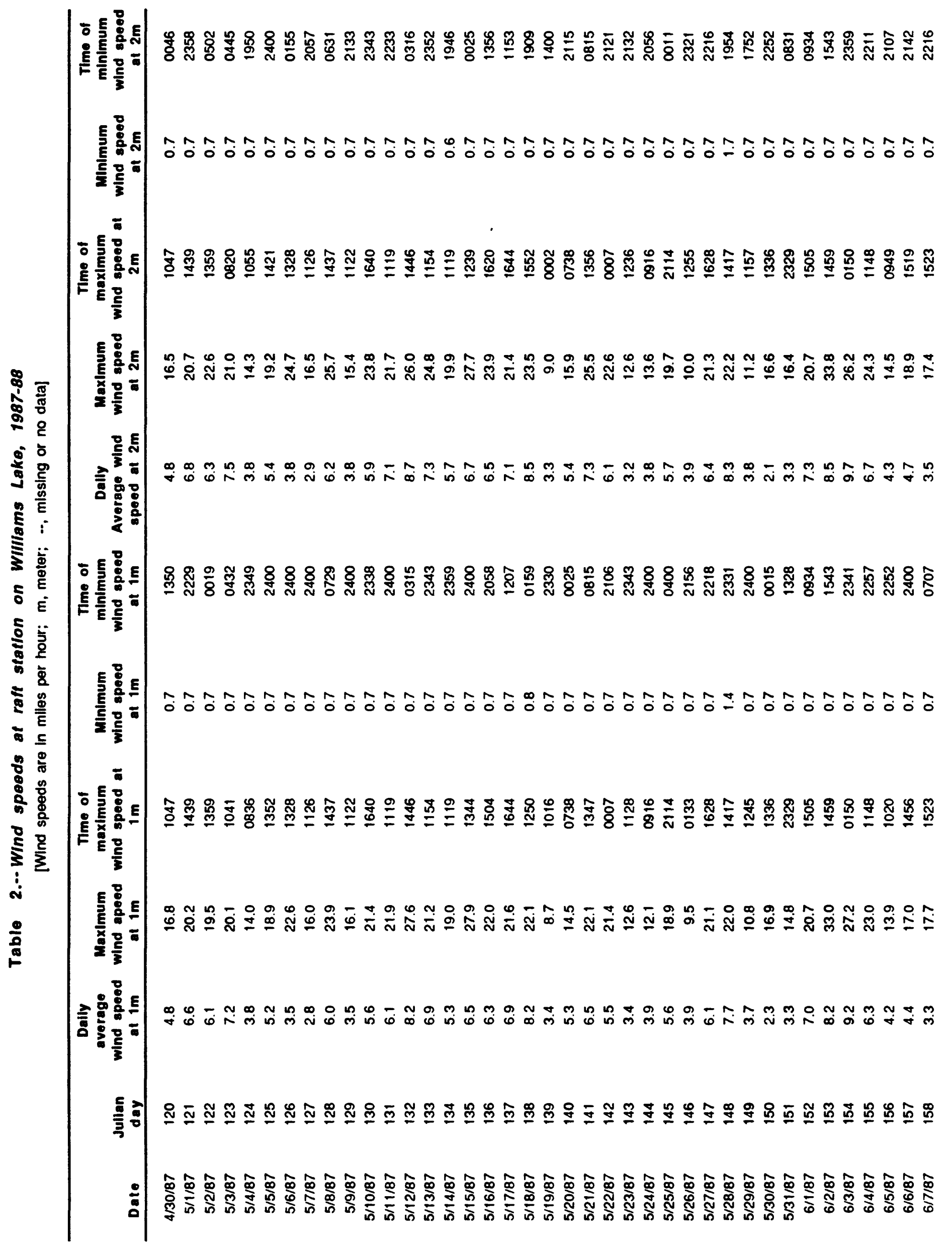




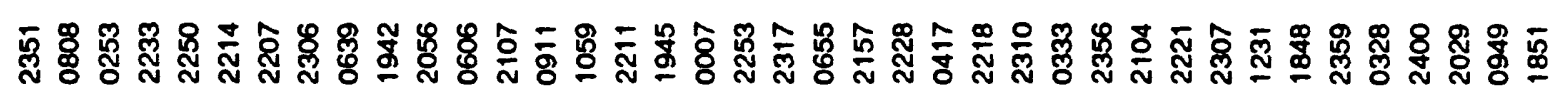
E 들

틀류 E

E

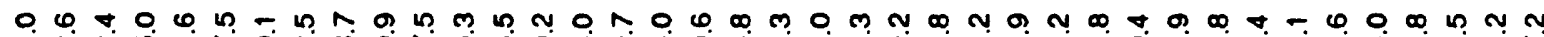

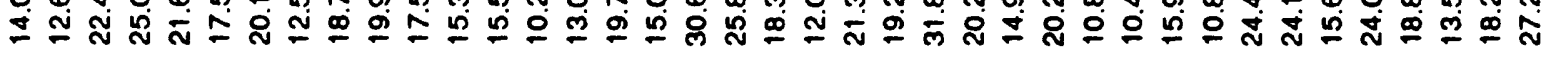
这 $\frac{1}{3}$

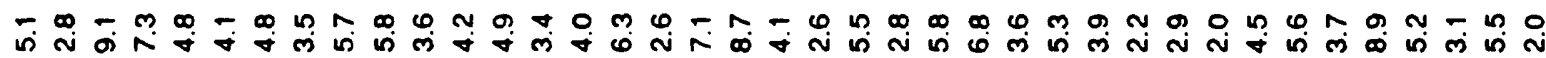

E

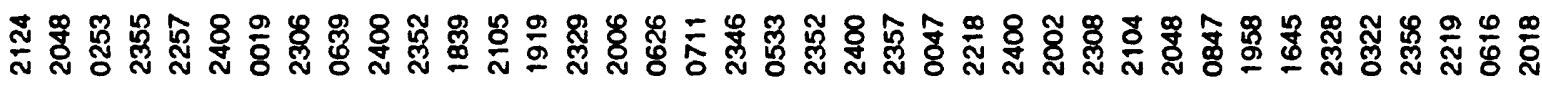

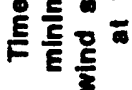
ส 


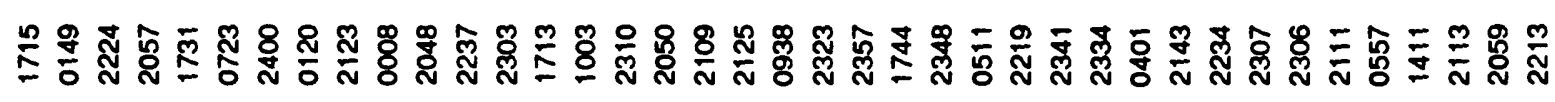

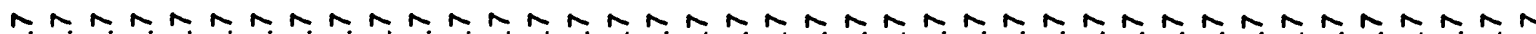
००

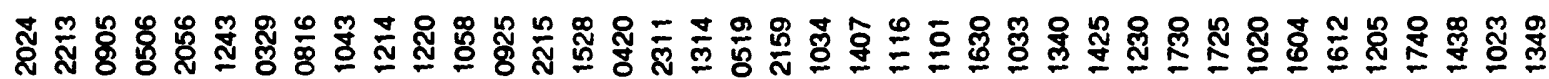

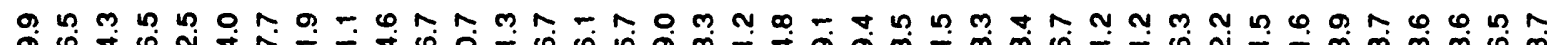
\&

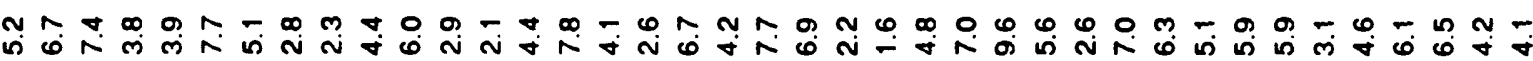

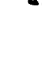

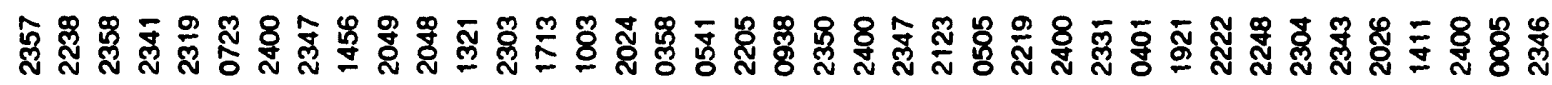

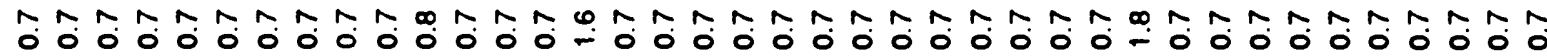

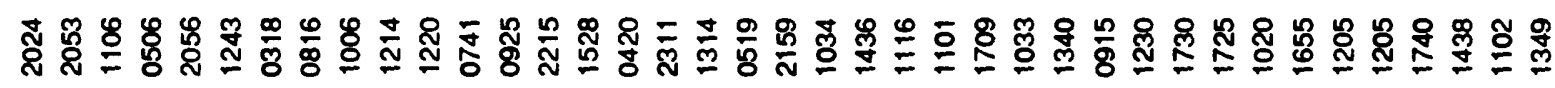

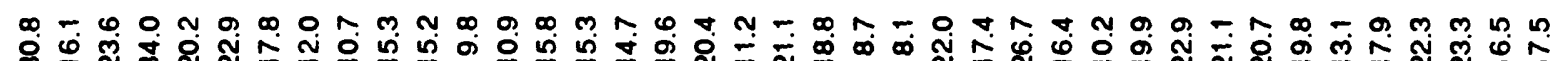
ำ

웁

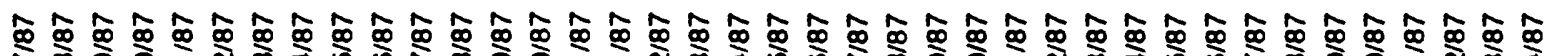

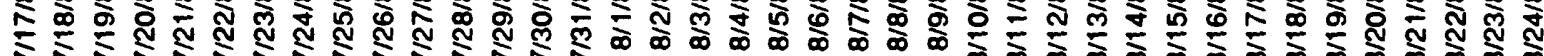

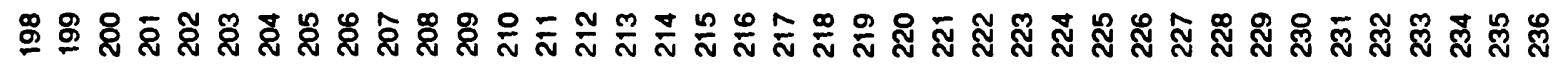

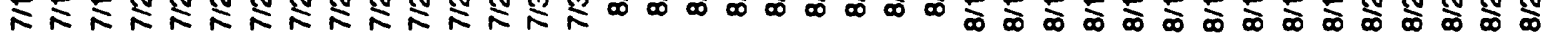




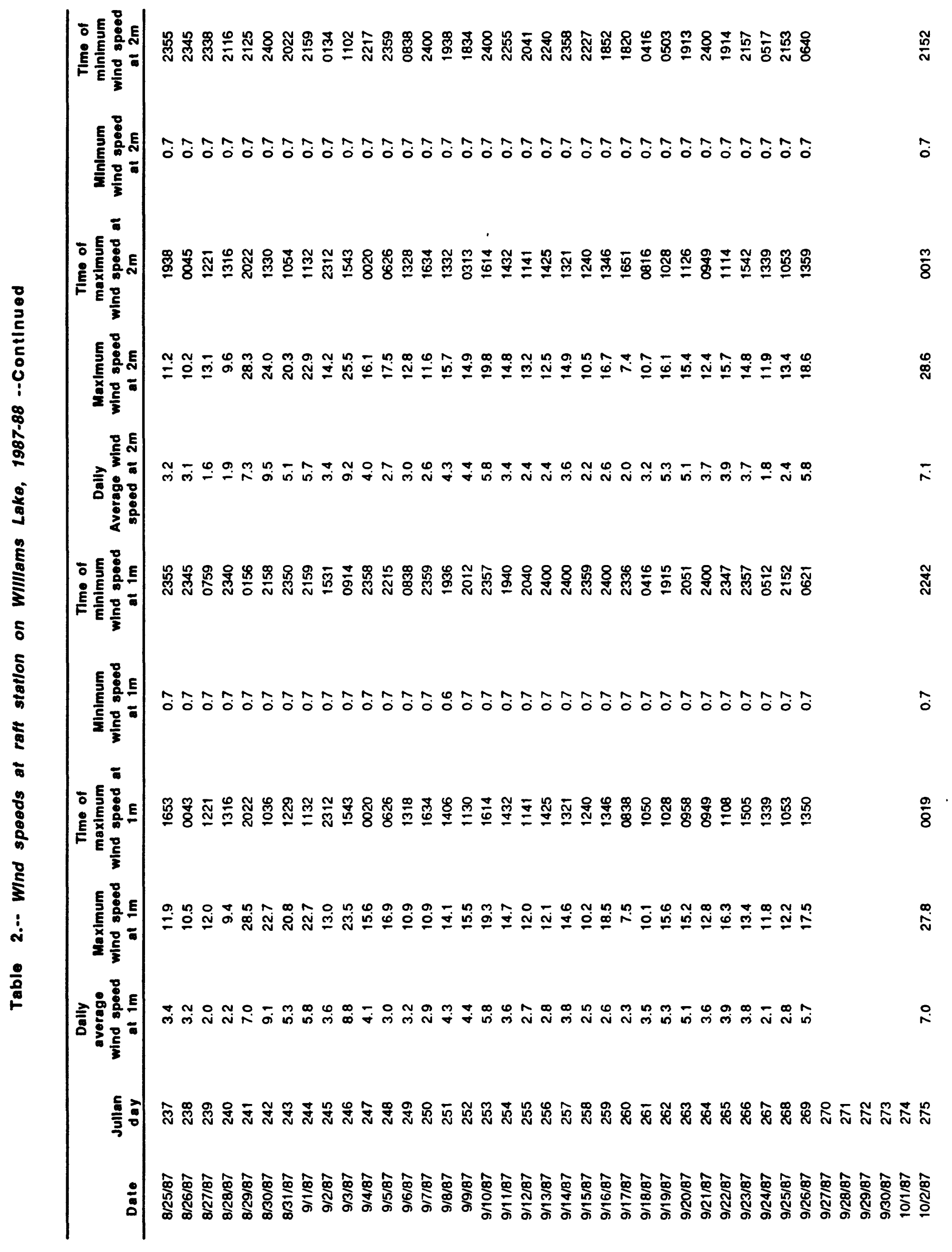




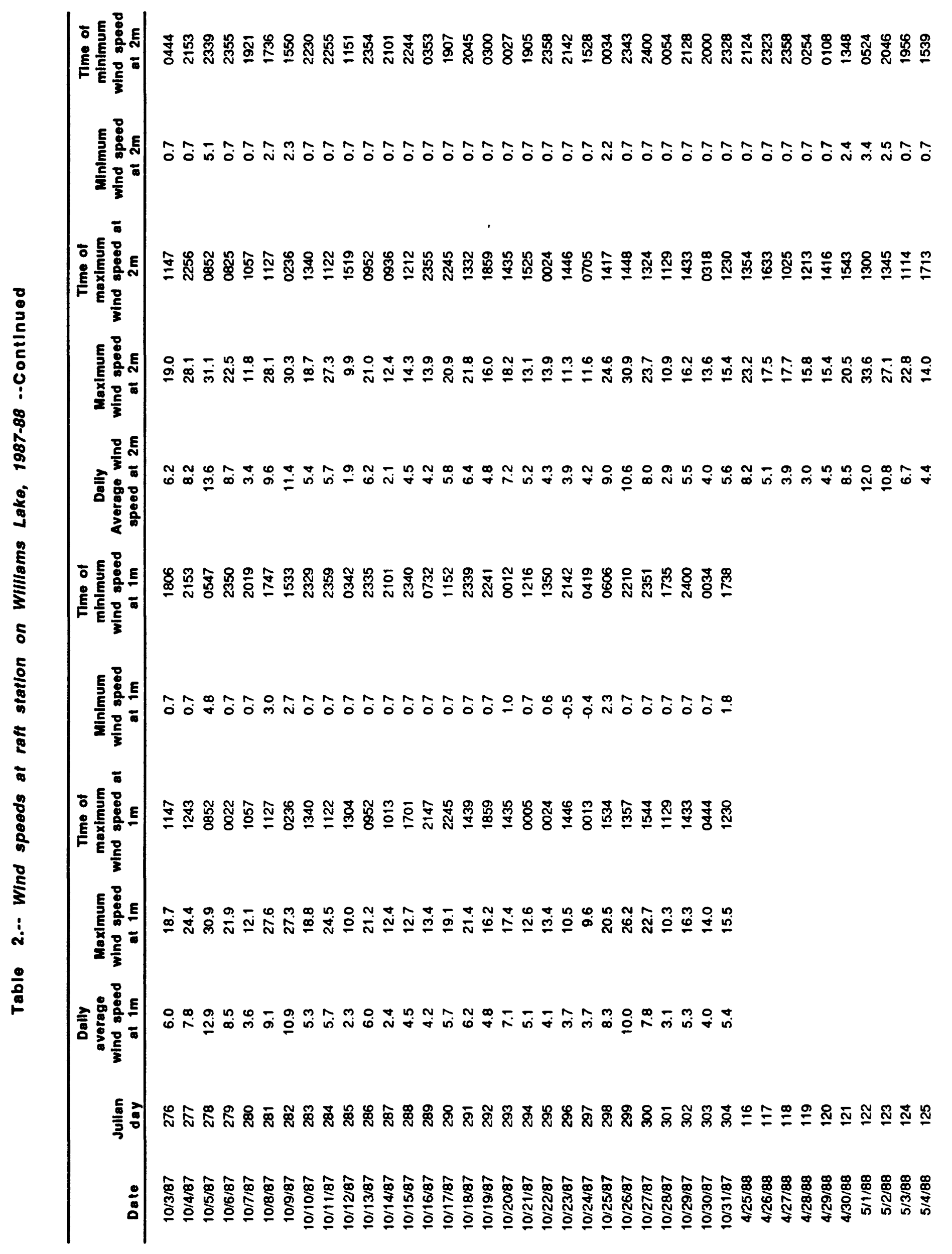




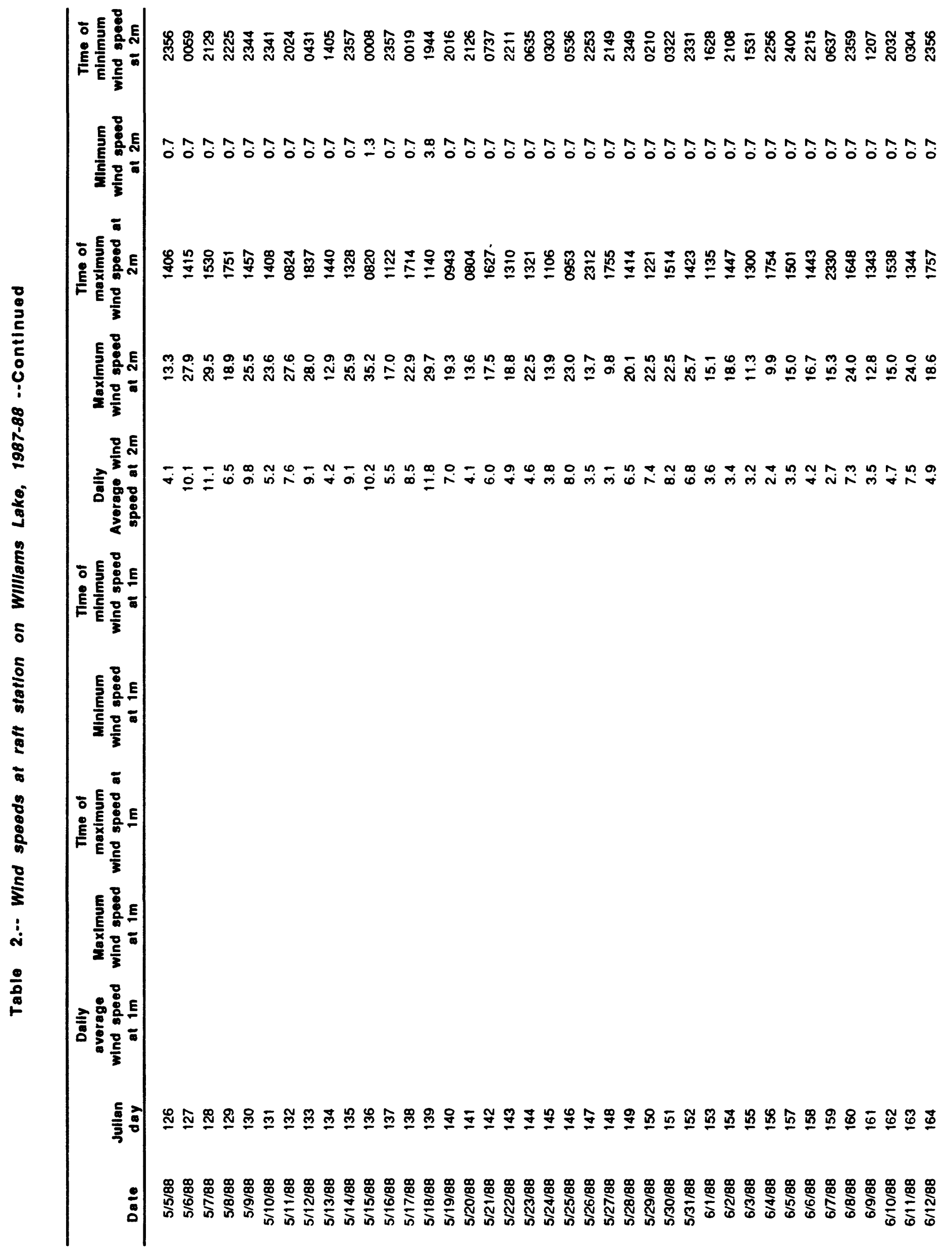




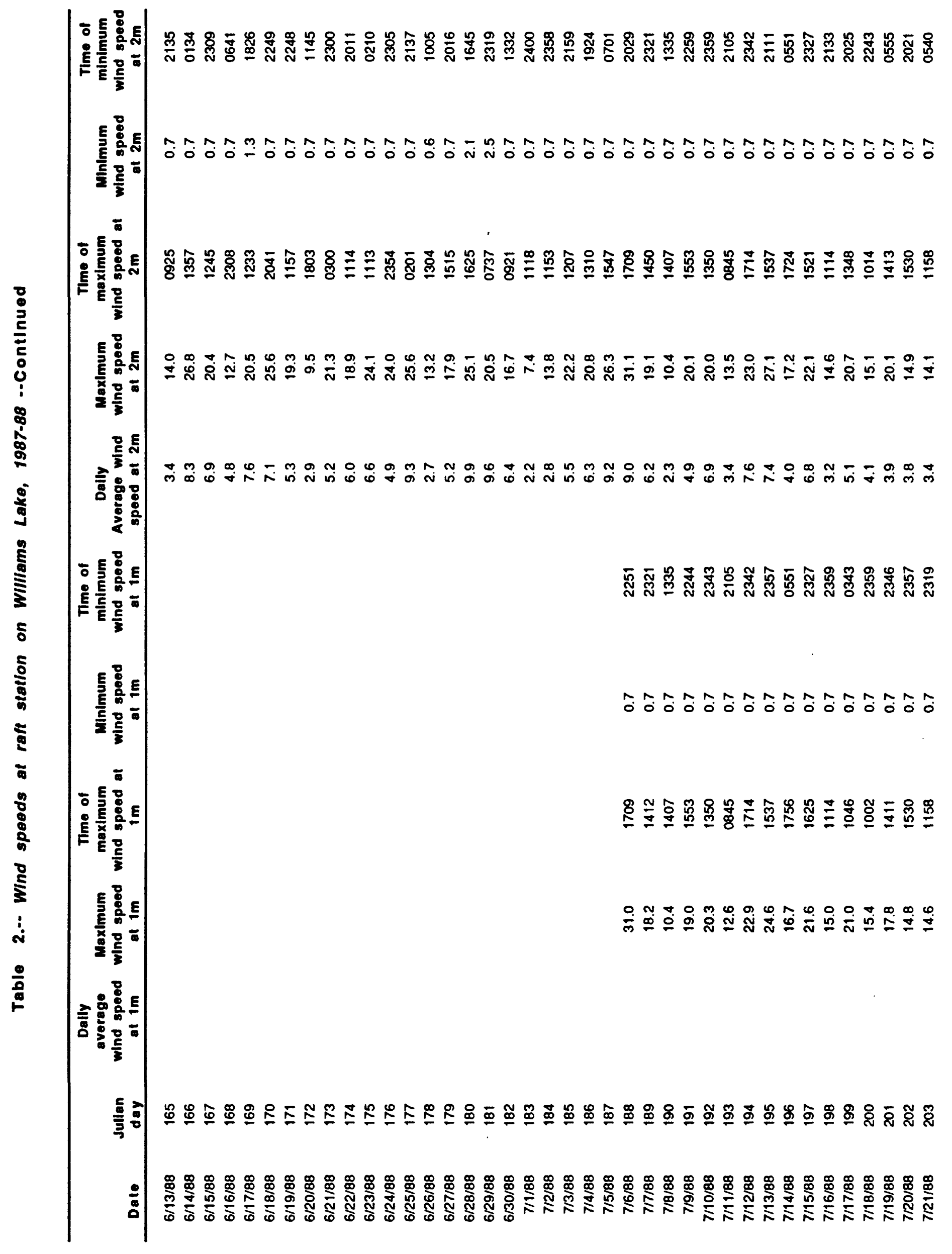




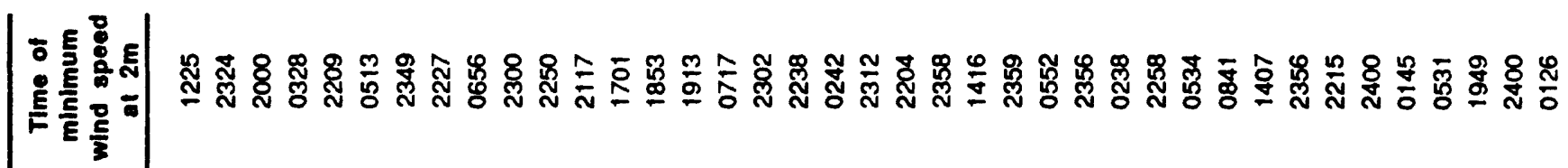

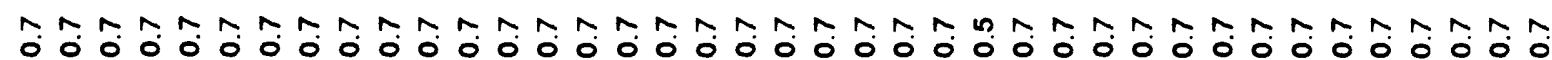

$\varphi-\nabla m m \forall n$ -

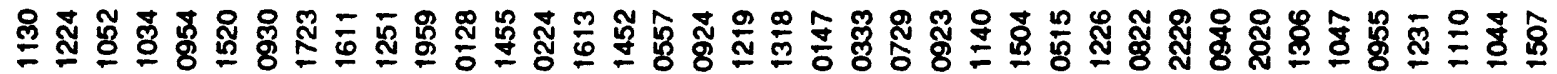

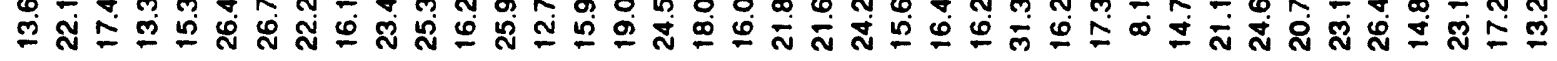

돈

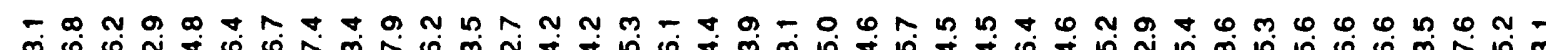

लं

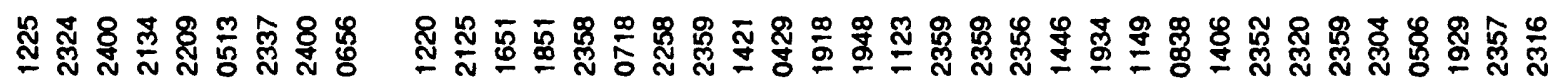

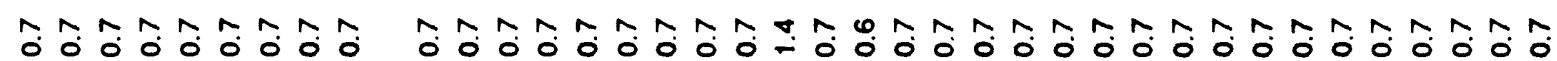

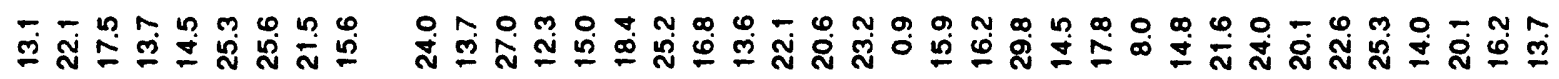

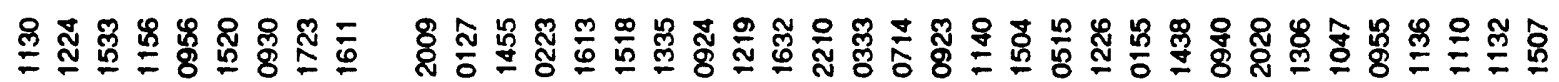

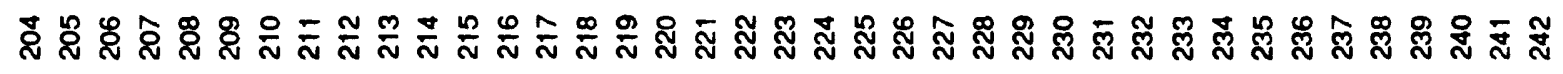




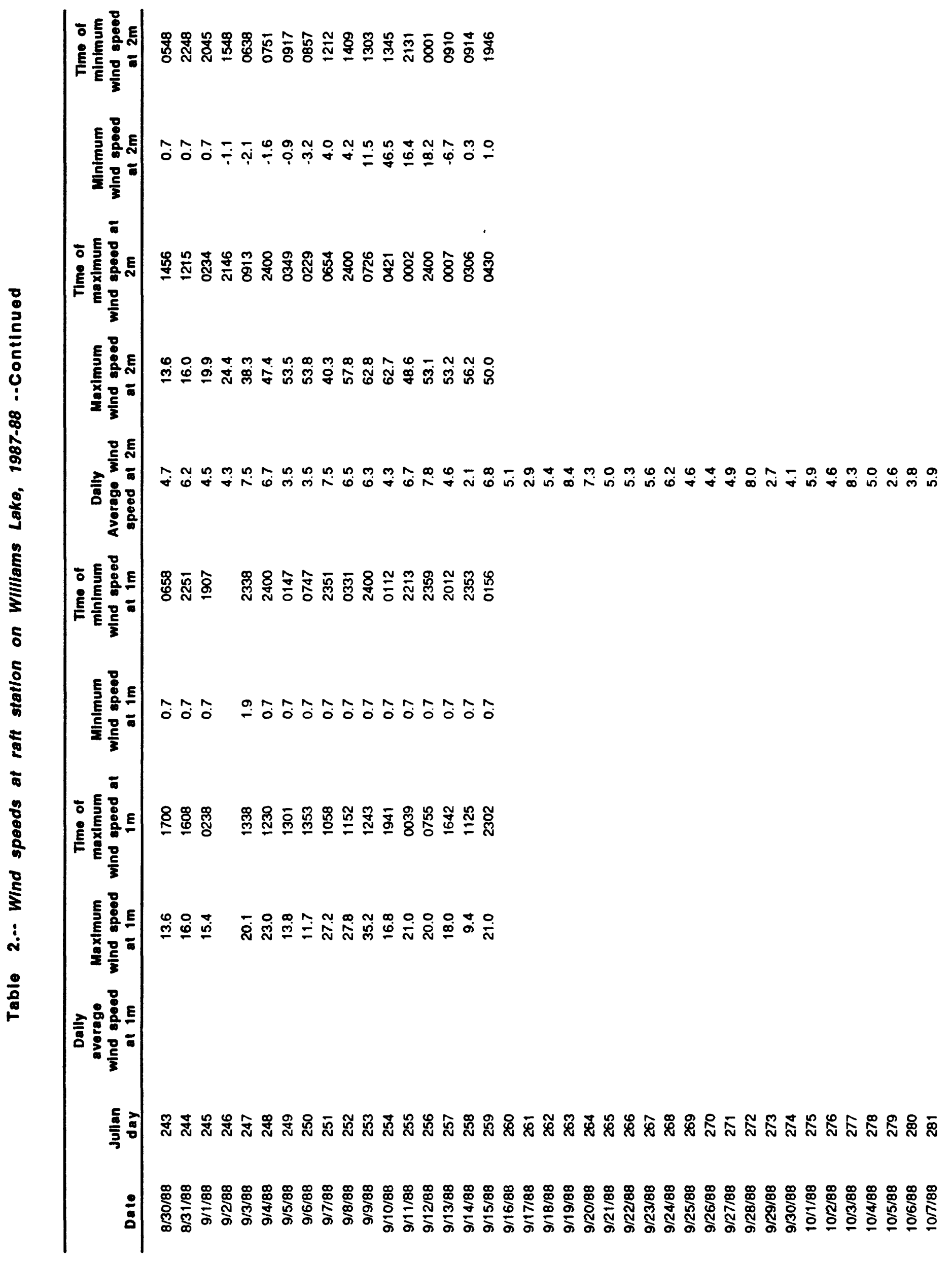




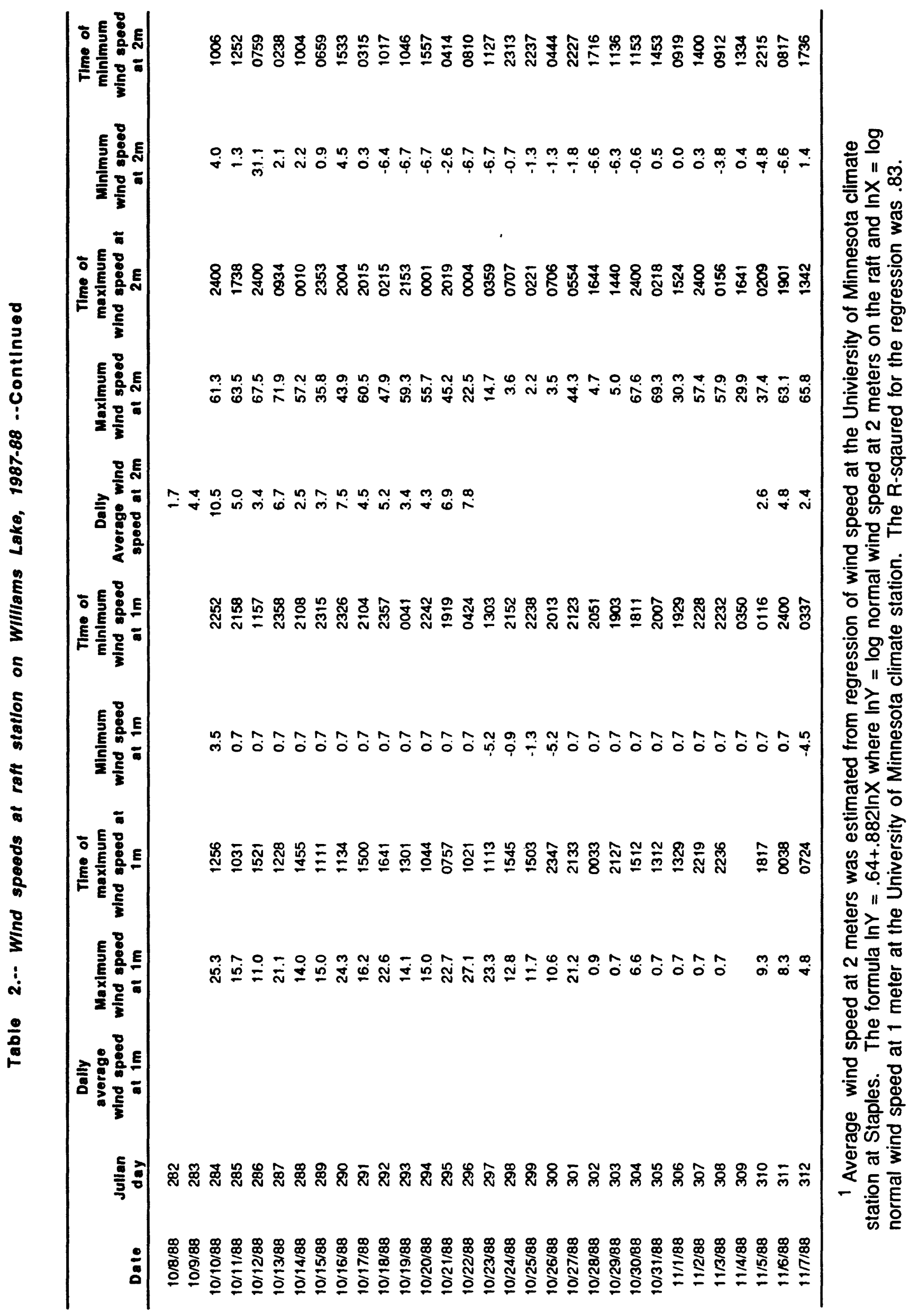


Table 3..-Precipitation for Wililams Lake gage and surrounding National Weather Service gages, 1987-88

[Precipitation measured in inches; NWS, National Weather Service; NWS average is a weighted average determined from the distance between the sites and Williams Lake; 0.00 , trace precipitation]

\begin{tabular}{|c|c|c|c|c|c|c|}
\hline Date & $\begin{array}{c}\text { Jullan } \\
\text { day }\end{array}$ & $\begin{array}{l}\text { Precipi- } \\
\text { tation at } \\
\text { Williame } \\
\text { Lake gauge }\end{array}$ & $\begin{array}{l}\text { NWS } \\
\text { precipita- } \\
\text { tion at Deep } \\
\text { Portage }\end{array}$ & $\begin{array}{l}\text { NWS } \\
\text { precipita- } \\
\text { tlon at Park } \\
\text { Raplds }\end{array}$ & $\begin{array}{l}\text { NWS } \\
\text { precipita. } \\
\text { tlon at } \\
\text { Pine River }\end{array}$ & $\begin{array}{l}\text { NWS } \\
\text { Precipl. } \\
\text { tatlon at } \\
\text { Walker }\end{array}$ \\
\hline $1 / 1 / 87$ & 1 & & & & & \\
\hline $1 / 2 / 87$ & 2 & & & & & \\
\hline $1 / 3 / 87$ & 3 & & & & & \\
\hline $1 / 4 / 87$ & 4 & & & & & \\
\hline $1 / 5 / 87$ & 5 & & & & & \\
\hline $1 / 6 / 87$ & 6 & & ' & & & 0.01 \\
\hline $1 / 7 / 87$ & 7 & & & & & \\
\hline $1 / 8 / 87$ & 8 & & & & & \\
\hline $1 / 9 / 87$ & 9 & & & 0.00 & & 0.00 \\
\hline $1 / 10 / 87$ & 10 & & & & & \\
\hline $1 / 11 / 87$ & 11 & & & & & \\
\hline $1 / 12 / 87$ & 12 & & & & & \\
\hline $1 / 13 / 87$ & 13 & & & & & \\
\hline $1 / 14 / 87$ & 14 & & & & & \\
\hline $1 / 15 / 87$ & 15 & & & & & 0.00 \\
\hline $1 / 16 / 87$ & 16 & & & & & \\
\hline $1 / 17 / 87$ & 17 & & & 0.00 & & \\
\hline $1 / 18 / 87$ & 18 & & & & & \\
\hline $1 / 19 / 87$ & 19 & & 0.88 & 0.10 & & 0.03 \\
\hline $1 / 20 / 87$ & 20 & & 0.22 & 0.03 & & 0.00 \\
\hline $1 / 21 / 87$ & 21 & & 0.44 & 0.01 & & 0.01 \\
\hline $1 / 22 / 87$ & 22 & & 0.00 & 0.00 & & \\
\hline $1 / 23 / 87$ & 23 & & & & & \\
\hline $1 / 24 / 87$ & 24 & & & & & \\
\hline $1 / 25 / 87$ & 25 & & & & & \\
\hline $1 / 26 / 87$ & 26 & & & & & 0.00 \\
\hline $1 / 27 / 87$ & 27 & & & 0.02 & & 0.00 \\
\hline $1 / 28 / 87$ & 28 & & & & & 0.00 \\
\hline $1 / 29 / 87$ & 29 & & 0.55 & 0.12 & & 0.18 \\
\hline $1 / 30 / 87$ & 30 & & 0.33 & 0.00 & & \\
\hline 1/31/87 & 31 & & & & & \\
\hline $2 / 1 / 87$ & 32 & & & & & \\
\hline $2 / 2 / 87$ & 33 & & & 0.18 & & 0.08 \\
\hline $2 / 3 / 87$ & 34 & & & & & \\
\hline $2 / 4 / 87$ & 35 & & & & & \\
\hline $2 / 5 / 87$ & 36 & & . & & & \\
\hline $2 / 6 / 87$ & 37 & & & & & \\
\hline $2 / 7 / 87$ & 38 & & & & & \\
\hline $2 / 8 / 87$ & 39 & & & & & \\
\hline 2/9/87 & 40 & & & & & \\
\hline $2 / 10 / 87$ & 41 & & & & & \\
\hline $2 / 11 / 87$ & 42 & & 0.80 & & & \\
\hline $2 / 12 / 87$ & 43 & & & & & \\
\hline $2 / 13 / 87$ & 44 & & & & & \\
\hline $2 / 14 / 87$ & 45 & & 0.00 & & & \\
\hline $2 / 15 / 87$ & 46 & & & & & \\
\hline $2 / 16 / 87$ & 47 & & & 0.03 & & . \\
\hline $2 / 17 / 87$ & 48 & & & & & \\
\hline $2 / 18 / 87$ & 49 & & & & & \\
\hline $2 / 19 / 87$ & 50 & & & & & \\
\hline $2 / 20 / 87$ & 51 & & & 0.00 & & \\
\hline $2 / 21 / 87$ & 52 & & & 0.11 & & 0.06 \\
\hline $2 / 22 / 87$ & 53 & & & 0.02 & & 0.03 \\
\hline $2 / 23 / 87$ & 54 & & 0.14 & 0.04 & & 0.00 \\
\hline $2 / 24 / 87$ & 55 & & & 0.21 & & 0.00 \\
\hline
\end{tabular}


Table 3..-Precipitation for Williams Lake gage and surrounding National Weather Service gages, 1987-88 -.Continued

\begin{tabular}{|c|c|c|c|c|c|c|}
\hline Date & $\begin{array}{c}\text { Julian } \\
\text { day }\end{array}$ & $\begin{array}{l}\text { Precipl- } \\
\text { tatlon at } \\
\text { WIIIlams } \\
\text { Lake gauge }\end{array}$ & $\begin{array}{l}\text { NWS } \\
\text { preciplta- } \\
\text { tion at Deep } \\
\text { Portage }\end{array}$ & $\begin{array}{l}\text { NWS } \\
\text { preciplta- } \\
\text { tlon at Park } \\
\text { Rapids }\end{array}$ & $\begin{array}{l}\text { NWS } \\
\text { precipita- } \\
\text { tion at } \\
\text { PIne River }\end{array}$ & $\begin{array}{l}\text { NWS } \\
\text { Preclpl- } \\
\text { tation at } \\
\text { Walker }\end{array}$ \\
\hline $2 / 25 / 87$ & 56 & & & & & \\
\hline $2 / 26 / 87$ & 57 & & & & & \\
\hline $2 / 27 / 87$ & 58 & & & & & \\
\hline 2/28/87 & 59 & & & 0.00 & & \\
\hline $3 / 1 / 87$ & 60 & & 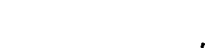 & 0.12 & & 0.18 \\
\hline $3 / 2 / 87$ & 61 & & & 0.00 & & \\
\hline $3 / 3 / 87$ & 62 & & & & & \\
\hline $3 / 4 / 87$ & 63 & & & & & \\
\hline $3 / 5 / 87$ & 64 & & & & & \\
\hline $3 / 6 / 87$ & 65 & & & & & \\
\hline $3 / 7 / 87$ & 66 & & & & & \\
\hline $3 / 8 / 87$ & 67 & & & & & \\
\hline $3 / 9 / 87$ & 68 & & & & & \\
\hline $3 / 10 / 87$ & 69 & & & & & \\
\hline $3 / 11 / 87$ & 70 & & & 0.00 & & 0.01 \\
\hline $3 / 12 / 87$ & 71 & & & & & \\
\hline $3 / 13 / 87$ & 72 & & & 0.04 & & 0.05 \\
\hline $3 / 14 / 87$ & 73 & & & & & 0.02 \\
\hline $3 / 15 / 87$ & 74 & & & & & \\
\hline $3 / 16 / 87$ & 75 & & & 0.00 & & \\
\hline $3 / 17 / 87$ & 76 & & & & & \\
\hline $3 / 18 / 87$ & 77 & & & & & \\
\hline $3 / 19 / 87$ & 78 & & & 0.00 & & 0.00 \\
\hline $3 / 20 / 87$ & 79 & & & & & \\
\hline $3 / 21 / 87$ & 80 & & & 0.07 & & 0.00 \\
\hline $3 / 22 / 87$ & 81 & & & 0.10 & & \\
\hline $3 / 23 / 87$ & 82 & & & 0.98 & & 0.85 \\
\hline $3 / 24 / 87$ & 83 & & 1.00 & 0.00 & & 0.22 \\
\hline $3 / 25 / 87$ & 84 & & & 0.00 & & \\
\hline $3 / 26 / 87$ & 85 & & & & & 0.01 \\
\hline $3 / 27 / 87$ & 86 & & & & & 0.00 \\
\hline $3 / 28 / 87$ & 87 & & & & & \\
\hline $3 / 29 / 87$ & 88 & & & 0.00 & & \\
\hline $3 / 30 / 87$ & 89 & & & & & \\
\hline $3 / 31 / 87$ & 90 & & & 0.00 & & 0.02 \\
\hline $4 / 1 / 87$ & 91 & & & 0.04 & & 0.06 \\
\hline $4 / 2 / 87$ & 92 & & & & & \\
\hline $4 / 3 / 87$ & 93 & & & & & \\
\hline $4 / 4 / 87$ & 94 & & & & & \\
\hline $4 / 5 / 87$ & 95 & & & & & \\
\hline $4 / 6 / 87$ & 96 & & & & & \\
\hline $4 / 7 / 87$ & 97 & & & & & \\
\hline $4 / 8 / 87$ & 98 & & & & & \\
\hline $4 / 9 / 87$ & 99 & & & & & \\
\hline $4 / 10 / 87$ & 100 & & & 0.00 & & \\
\hline 4/11/87 & 101 & & & & & \\
\hline 4/12/87 & 102 & & & & & \\
\hline 4/13/87 & 103 & & & 0.00 & & 0.00 \\
\hline $4 / 14 / 87$ & 104 & & & & & \\
\hline $4 / 15 / 87$ & 105 & & & & & \\
\hline $4 / 16 / 87$ & 106 & & & & & \\
\hline $4 / 17 / 87$ & 107 & & & & & \\
\hline $4 / 18 / 87$ & 108 & & & & & \\
\hline $4 / 19 / 87$ & 109 & & & & & \\
\hline 4/20/87 & 110 & & & 0.15 & & 0.14 \\
\hline
\end{tabular}


Table 3..-Preclpitation for Williams Lake gage and surrounding National Weather Service gages, 1987-88 -.Continued

\begin{tabular}{|c|c|c|c|c|c|c|}
\hline Date & $\begin{array}{c}\text { Jullan } \\
\text { day }\end{array}$ & $\begin{array}{l}\text { Precipl- } \\
\text { tation at } \\
\text { Williams } \\
\text { Lake gauge }\end{array}$ & $\begin{array}{l}\text { NWS } \\
\text { precipita- } \\
\text { tion at Deep } \\
\text { Portage }\end{array}$ & $\begin{array}{l}\text { NWS } \\
\text { Preclpita- } \\
\text { tlon at Park } \\
\text { Rapids }\end{array}$ & $\begin{array}{l}\text { NWS } \\
\text { preciplta- } \\
\text { tion at } \\
\text { PIne River }\end{array}$ & $\begin{array}{l}\text { NWS } \\
\text { Precipl- } \\
\text { tation at } \\
\text { Walker }\end{array}$ \\
\hline $4 / 21 / 87$ & 111 & & & & & \\
\hline $4 / 22 / 87$ & 112 & & & & & \\
\hline $4 / 23 / 87$ & 113 & & & & & \\
\hline $4 / 24 / 87$ & 114 & & & & & \\
\hline $4 / 25 / 87$ & 115 & & . & & & 0.00 \\
\hline $4 / 26 / 87$ & 116 & & & 0.00 & & 0.10 \\
\hline $4 / 27 / 87$ & 117 & & & & & \\
\hline $4 / 28 / 87$ & 118 & & & & & \\
\hline $4 / 29 / 87$ & 119 & & & & & \\
\hline $4 / 30 / 87$ & 120 & 0.02 & & 0.00 & & 0.19 \\
\hline $5 / 1 / 87$ & 121 & & & & & 0.06 \\
\hline $5 / 2 / 87$ & 122 & & & & & \\
\hline $5 / 3 / 87$ & 123 & & & & & \\
\hline $5 / 4 / 87$ & 124 & & & & & \\
\hline $5 / 5 / 87$ & 125 & & & & & \\
\hline $5 / 6 / 87$ & 126 & & & & & \\
\hline $5 / 7 / 87$ & 127 & & & & & \\
\hline $5 / 8 / 87$ & 128 & & & & & \\
\hline $5 / 9 / 87$ & 129 & & & & & \\
\hline $5 / 10 / 87$ & 130 & & & 0.04 & & 0.02 \\
\hline $5 / 11 / 87$ & 131 & & 0.02 & & & \\
\hline $5 / 12 / 87$ & 132 & & & 0.00 & & 0.01 \\
\hline $5 / 13 / 87$ & 133 & 0.30 & & 0.60 & & 0.33 \\
\hline $5 / 14 / 87$ & 134 & & 0.17 & & & \\
\hline $5 / 15 / 87$ & 135 & 0.09 & & 0.07 & & 0.08 \\
\hline $5 / 16 / 87$ & 136 & 0.20 & 0.05 & 0.00 & & \\
\hline $5 / 17 / 87$ & 137 & 1.75 & & 0.73 & & 2.07 \\
\hline $5 / 18 / 87$ & 138 & 0.05 & 1.90 & 0.64 & & 0.76 \\
\hline $5 / 19 / 87$ & 139 & & 0.06 & 0.04 & & 0.04 \\
\hline $5 / 20 / 87$ & 140 & 0.93 & 0.00 & 0.05 & & 0.21 \\
\hline $5 / 21 / 87$ & 141 & 1.13 & 1.55 & 1.27 & & 1.65 \\
\hline $5 / 22 / 87$ & 142 & 0.31 & & 0.26 & & 0.69 \\
\hline $5 / 23 / 87$ & 143 & & 0.01 & & & \\
\hline $5 / 24 / 87$ & 144 & & & 0.00 & & 0.00 \\
\hline $5 / 25 / 87$ & 145 & 0.49 & 0.01 & 0.73 & & 0.32 \\
\hline $5 / 26 / 87$ & 146 & 0.03 & 0.28 & 0.26 & & 0.19 \\
\hline $5 / 27 / 87$ & 147 & 0.08 & 0.00 & 0.03 & & 0.10 \\
\hline $5 / 28 / 87$ & 148 & & & & & \\
\hline $5 / 29 / 87$ & 149 & 0.02 & & 0.70 & & \\
\hline $5 / 30 / 87$ & 150 & 0.36 & 0.49 & & & 0.05 \\
\hline $5 / 31 / 87$ & 151 & & & & & 0.00 \\
\hline $6 / 1 / 87$ & 152 & 0.17 & & 0.78 & & 0.18 \\
\hline $6 / 2 / 87$ & 153 & 0.09 & & 0.02 & & 0.30 \\
\hline $6 / 3 / 87$ & 154 & & & & & \\
\hline $6 / 4 / 87$ & 155 & & & & & \\
\hline $6 / 5 / 87$ & 156 & & & & & \\
\hline $6 / 6 / 87$ & 157 & & & & & \\
\hline $6 / 7 / 87$ & 158 & & & 0.00 & & \\
\hline $6 / 8 / 87$ & 159 & & & 0.00 & & \\
\hline $6 / 9 / 87$ & 160 & & & & & \\
\hline $6 / 10 / 87$ & 161 & 0.17 & & 0.10 & & 0.26 \\
\hline $6 / 11 / 87$ & 162 & & 0.65 & 0.00 & & 0.00 \\
\hline $6 / 12 / 87$ & 163 & & & & & \\
\hline $6 / 13 / 87$ & 164 & & & & & \\
\hline $6 / 14 / 87$ & 165 & & & & & \\
\hline
\end{tabular}


Table 3... Precipitation for Williams Lake gage and surrounding National Weather Service gages, 1987-88 --Continued

\begin{tabular}{|c|c|c|c|c|c|c|}
\hline Date & $\begin{array}{c}\text { Jullan } \\
\text { day }\end{array}$ & $\begin{array}{l}\text { Preclpl- } \\
\text { tation at } \\
\text { Williams } \\
\text { Lake gauge }\end{array}$ & $\begin{array}{l}\text { NWS } \\
\text { preclplta- } \\
\text { tlon at Deep } \\
\text { Portage }\end{array}$ & $\begin{array}{l}\text { NWS } \\
\text { preclplta- } \\
\text { tlon at Park } \\
\text { Raplds }\end{array}$ & $\begin{array}{l}\text { NWS } \\
\text { precipita- } \\
\text { tlon at } \\
\text { PIne River }\end{array}$ & $\begin{array}{l}\text { NWS } \\
\text { Precipl. } \\
\text { tation at } \\
\text { Walker }\end{array}$ \\
\hline $6 / 15 / 87$ & 166 & & & & & \\
\hline $6 / 16 / 87$ & 167 & & & & & \\
\hline $6 / 17 / 87$ & 168 & 0.01 & & 0.09 & & 0.00 \\
\hline $6 / 18 / 87$ & 169 & & & & & \\
\hline $6 / 19 / 87$ & 170 & & & & & \\
\hline $6 / 20 / 87$ & 171 & & . & & & \\
\hline $6 / 21 / 87$ & 172 & & & & & \\
\hline $6 / 22 / 87$ & 173 & & & 0.00 & & \\
\hline $6 / 23 / 87$ & 174 & 0.15 & & 0.10 & & 0.47 \\
\hline $6 / 24 / 87$ & 175 & & & 0.00 & & 0.29 \\
\hline $6 / 25 / 87$ & 176 & & & & & 0.00 \\
\hline $6 / 26 / 87$ & 177 & & & 0.00 & & 0.01 \\
\hline $6 / 27 / 87$ & 178 & & & & & \\
\hline $6 / 28 / 87$ & 179 & 0.01 & & 0.08 & & \\
\hline $6 / 29 / 87$ & 180 & 0.10 & & 0.06 & & 0.03 \\
\hline $6 / 30 / 87$ & 181 & & & & & \\
\hline $7 / 1 / 87$ & 182 & 0.10 & & 0.10 & & 0.30 \\
\hline $7 / 2 / 87$ & 183 & 0.05 & & 0.00 & & 0.47 \\
\hline $7 / 3 / 87$ & 184 & & & & & \\
\hline $7 / 4 / 87$ & 185 & & & & & \\
\hline $7 / 5 / 87$ & 186 & 0.26 & 0.18 & 0.12 & & 0.13 \\
\hline $7 / 6 / 87$ & 187 & & 0.00 & & & 0.01 \\
\hline $7 / 7 / 87$ & 188 & & & & & 0.00 \\
\hline $7 / 8 / 87$ & 189 & & & & & \\
\hline $7 / 9 / 87$ & 190 & & & & & \\
\hline $7 / 10 / 87$ & 191 & 0.35 & & 0.00 & & \\
\hline $7 / 11 / 87$ & 192 & 0.03 & 0.78 & 0.88 & & 0.30 \\
\hline $7 / 12 / 87$ & 193 & 0.03 & 0.10 & 0.04 & & 0.03 \\
\hline $7 / 13 / 87$ & 194 & & 0.00 & & & \\
\hline $7 / 14 / 87$ & 195 & 0.03 & & & & \\
\hline $7 / 15 / 87$ & 196 & 0.05 & 0.13 & 0.24 & & 0.06 \\
\hline $7 / 16 / 87$ & 197 & & & & & \\
\hline $7 / 17 / 87$ & 198 & 1.01 & & 0.28 & & 0.27 \\
\hline $7 / 18 / 87$ & 199 & 0.40 & 1.12 & 1.00 & & 1.65 \\
\hline $7 / 19 / 87$ & 200 & 0.62 & 1.48 & 0.80 & & 0.95 \\
\hline $7 / 20 / 87$ & 201 & 0.53 & 0.35 & 0.48 & & 0.45 \\
\hline $7 / 21 / 87$ & 202 & 0.77 & 0.02 & & & \\
\hline $7 / 22 / 87$ & 203 & & 0.06 & 1.33 & & 0.72 \\
\hline $7 / 23 / 87$ & 204 & 0.57 & 0.40 & 0.29 & & 0.97 \\
\hline $7 / 24 / 87$ & 205 & & & & & \\
\hline $7 / 25 / 87$ & 206 & 0.01 & & 0.00 & & 0.01 \\
\hline $7 / 26 / 87$ & 207 & & & 0.00 & & \\
\hline $7 / 27 / 87$ & 208 & & & & & \\
\hline $7 / 28 / 87$ & 209 & & & & & \\
\hline $7 / 29 / 87$ & 210 & & & & & \\
\hline $7 / 30 / 87$ & 211 & & & & & \\
\hline $7 / 31 / 87$ & 212 & 0.01 & 0.02 & 0.00 & & 0.03 \\
\hline $8 / 4 / 87$ & 213 & 0.01 & & 0.02 & & 0.02 \\
\hline $8 / 2 / 87$ & 214 & 0.04 & & & & \\
\hline $8 / 3 / 87$ & 215 & & 0.33 & 0.29 & & 0.13 \\
\hline $8 / 4 / 87$ & 216 & & & & & \\
\hline $8 / 5 / 87$ & 217 & 0.02 & & & & \\
\hline $8 / 6 / 87$ & 218 & & 0.01 & & & 0.11 \\
\hline $8 / 7 / 87$ & 219 & & & & & \\
\hline $8 / 8 / 87$ & 220 & & & & & \\
\hline
\end{tabular}


Table 3...Precipitation for Wilitims Lake gage and surrounding National Weather Service gages, $1987-88$--Continued

\begin{tabular}{|c|c|c|c|c|c|c|}
\hline Date & $\begin{array}{c}\text { Jullan } \\
\text { day }\end{array}$ & $\begin{array}{l}\text { Precipl. } \\
\text { tation at } \\
\text { Williams } \\
\text { Lake gauge }\end{array}$ & $\begin{array}{l}\text { NWS } \\
\text { preclpita- } \\
\text { tion at Deep } \\
\text { Portage }\end{array}$ & $\begin{array}{l}\text { NWS } \\
\text { preclpita- } \\
\text { tion at Park } \\
\text { Rapids }\end{array}$ & $\begin{array}{l}\text { NWS } \\
\text { precipita- } \\
\text { tion at } \\
\text { Pine River }\end{array}$ & $\begin{array}{l}\text { NWS } \\
\text { Precipl- } \\
\text { tation at } \\
\text { Walker }\end{array}$ \\
\hline $8 / 9 / 87$ & 221 & & & & & \\
\hline $8 / 10 / 87$ & 222 & & & & & \\
\hline $8 / 11 / 87$ & 223 & & & & & \\
\hline $8 / 12 / 87$ & 224 & 0.10 & 0.03 & 0.20 & & 0.58 \\
\hline $8 / 13 / 87$ & 225 & & 0.00 & & & \\
\hline $8 / 14 / 87$ & 226 & & & & & \\
\hline $8 / 15 / 87$ & 227 & 1.23 & 0.33 & 0.47 & & 0.23 \\
\hline $8 / 16 / 87$ & 228 & & 1.58 & 1.90 & & 1.24 \\
\hline $8 / 17 / 87$ & 229 & 0.15 & 0.00 & 0.07 & & 0.19 \\
\hline $8 / 18 / 87$ & 230 & 0.05 & 0.13 & 0.32 & & 0.07 \\
\hline $8 / 19 / 87$ & 231 & & & & & \\
\hline $8 / 20 / 87$ & 232 & & & & & 0.01 \\
\hline $8 / 21 / 87$ & 233 & 0.03 & & 0.21 & & 0.05 \\
\hline $8 / 22 / 87$ & 234 & & 0.02 & 0.04 & & \\
\hline $8 / 23 / 87$ & 235 & & & & & \\
\hline $8 / 24 / 87$ & 236 & & & & & \\
\hline $8 / 25 / 87$ & 237 & 0.17 & 0.00 & & & 0.10 \\
\hline $8 / 26 / 87$ & 238 & 0.14 & 0.14 & 0.12 & & 0.34 \\
\hline $8 / 27 / 87$ & 239 & 0.01 & 0.02 & & & \\
\hline $8 / 28 / 87$ & 240 & & & & & \\
\hline $8 / 29 / 87$ & 241 & 0.22 & & & & \\
\hline $8 / 30 / 87$ & 242 & & & 0.32 & & 0.24 \\
\hline $8 / 31 / 87$ & 243 & & & & & \\
\hline $9 / 1 / 87$ & 244 & & & & & 0.01 \\
\hline $9 / 2 / 87$ & 245 & & & & & \\
\hline $9 / 3 / 87$ & 246 & & & 0.00 & & \\
\hline $9 / 4 / 87$ & 247 & & & 0.00 & & 0.00 \\
\hline $9 / 5 / 87$ & 248 & & & 0.65 & & 0.76 \\
\hline $9 / 6 / 87$ & 249 & & & 0.00 & & 0.00 \\
\hline $9 / 7 / 87$ & 250 & 0.01 & & 0.00 & & 0.05 \\
\hline $9 / 8 / 87$ & 251 & 0.01 & & 0.02 & & 0.01 \\
\hline $9 / 9 / 87$ & 252 & 0.03 & & 0.12 & & 0.26 \\
\hline $9 / 10 / 87$ & 253 & 0.08 & & 0.00 & & 0.05 \\
\hline $9 / 11 / 87$ & 254 & & & & & 0.02 \\
\hline $9 / 12 / 87$ & 255 & 0.17 & & 0.25 & & 0.19 \\
\hline $9 / 13 / 87$ & 256 & & & 0.03 & & 0.07 \\
\hline $9 / 14 / 87$ & 257 & & & & & \\
\hline $9 / 15 / 87$ & 258 & & & & & \\
\hline $9 / 16 / 87$ & 259 & & & & & \\
\hline $9 / 17 / 87$ & 260 & 0.02 & & & & \\
\hline $9 / 18 / 87$ & 261 & 2.19 & & 2.36 & & 2.05 \\
\hline $9 / 19 / 87$ & 262 & 0.24 & & 0.38 & & 0.57 \\
\hline $9 / 20 / 87$ & 263 & 0.03 & & 0.08 & & 0.11 \\
\hline $9 / 21 / 87$ & 264 & & & & & \\
\hline $9 / 22 / 87$ & 265 & & & & & \\
\hline $9 / 23 / 87$ & 266 & & & & & \\
\hline $9 / 24 / 87$ & 267 & & & 0.02 & & \\
\hline $9 / 25 / 87$ & 268 & 0.17 & & & & \\
\hline $9 / 26 / 87$ & 269 & 0.14 & & & & \\
\hline $9 / 27 / 87$ & 270 & 0.01 & & & & \\
\hline $9 / 28 / 87$ & 271 & & & 0.00 & & \\
\hline $9 / 29 / 87$ & 272 & 0.22 & & 0.02 & & 0.13 \\
\hline $9 / 30 / 87$ & 273 & & & 0.02 & & \\
\hline $10 / 1 / 87$ & 274 & & & 0.01 & & 0.02 \\
\hline $10 / 2 / 87$ & 275 & & & & & \\
\hline
\end{tabular}


Table 3...Precipitation for Wililams Lake gage and surrounding National Weather Service gages, $1987-88$-.Continued

\begin{tabular}{|c|c|c|c|c|c|c|}
\hline Date & $\begin{array}{l}\text { Julian } \\
\text { day }\end{array}$ & $\begin{array}{l}\text { Precipl- } \\
\text { tation at } \\
\text { Williams } \\
\text { Lake gauge }\end{array}$ & $\begin{array}{l}\text { NWS } \\
\text { preciplta- } \\
\text { tion at Deep } \\
\text { Portage }\end{array}$ & $\begin{array}{l}\text { NWS } \\
\text { precipita- } \\
\text { tion at Park } \\
\text { Rapids }\end{array}$ & $\begin{array}{l}\text { NWS } \\
\text { precipita- } \\
\text { tion at } \\
\text { Pine River }\end{array}$ & $\begin{array}{l}\text { NWS } \\
\text { Precipl- } \\
\text { tation at } \\
\text { Walker }\end{array}$ \\
\hline $10 / 3 / 87$ & 276 & & & & & \\
\hline $10 / 4 / 87$ & 277 & & & & & \\
\hline $10 / 5 / 87$ & 278 & & & 0.04 & & 0.12 \\
\hline $10 / 6 / 87$ & 279 & & & 0.12 & & 0.01 \\
\hline $10 / 7 / 87$ & 280 & & & & & \\
\hline $10 / 8 / 87$ & 281 & & ${ }^{\prime}$ & & & \\
\hline $10 / 9 / 87$ & 282 & & & 0.00 & & \\
\hline $10 / 10 / 87$ & 283 & & & & & \\
\hline $10 / 11 / 87$ & 284 & & & & & \\
\hline $10 / 12 / 87$ & 285 & & & & & \\
\hline $10 / 13 / 87$ & 286 & & & & & \\
\hline $10 / 14 / 87$ & 287 & & & & & \\
\hline $10 / 15 / 87$ & 288 & 0.13 & & 0.14 & & 0.04 \\
\hline $10 / 16 / 87$ & 289 & 0.21 & 0.50 & 0.11 & & 0.14 \\
\hline $10 / 17 / 87$ & 290 & & 0.06 & 0.02 & & 0.10 \\
\hline $10 / 18 / 87$ & 291 & & & & & \\
\hline $10 / 19 / 87$ & 292 & & & & & \\
\hline $10 / 20 / 87$ & 293 & 0.08 & & 0.05 & & 0.07 \\
\hline $10 / 21 / 87$ & 294 & & & & & 0.00 \\
\hline $10 / 22 / 87$ & 295 & & 0.19 & 0.15 & & 0.10 \\
\hline $10 / 23 / 87$ & 296 & 0.07 & & 0.03 & & 0.00 \\
\hline $10 / 24 / 87$ & 297 & 0.10 & 0.22 & & & 0.00 \\
\hline $10 / 25 / 87$ & 298 & 0.18 & & 0.11 & & \\
\hline $10 / 26 / 87$ & 299 & & & & & \\
\hline $10 / 27 / 87$ & 300 & 0.02 & & 0.00 & & \\
\hline $10 / 28 / 87$ & 301 & & & 0.00 & & \\
\hline $10 / 29 / 87$ & 302 & & & & & \\
\hline $10 / 30 / 87$ & 303 & & & & & \\
\hline $10 / 31 / 87$ & 304 & & & & & \\
\hline $11 / 1 / 87$ & 305 & & & 0.00 & & \\
\hline $11 / 2 / 87$ & 306 & & & 0.02 & & 0.02 \\
\hline $11 / 3 / 87$ & 307 & & 0.16 & 0.05 & & 0.01 \\
\hline $11 / 4 / 87$ & 308 & & 0.03 & 0.03 & & 0.02 \\
\hline $11 / 5 / 87$ & 309 & & & & & \\
\hline $11 / 6 / 87$ & 310 & & & & & \\
\hline $11 / 7 / 87$ & 311 & & & & & \\
\hline $11 / 8 / 87$ & 312 & & & & & \\
\hline $11 / 9 / 87$ & 313 & & & & & \\
\hline $11 / 10 / 87$ & 314 & & & & & \\
\hline $11 / 11 / 87$ & 315 & & & & & \\
\hline $11 / 12 / 87$ & 316 & & & & & \\
\hline $11 / 13 / 87$ & 317 & & & & & \\
\hline $11 / 14 / 87$ & 318 & & & & & \\
\hline $11 / 15 / 87$ & 319 & & 0.10 & 0.15 & & 0.06 \\
\hline $11 / 16 / 87$ & 320 & & 2.10 & 0.74 & & 0.65 \\
\hline $11 / 17 / 87$ & 321 & & & 0.10 & & \\
\hline $11 / 18 / 87$ & 322 & & & & & \\
\hline $11 / 19 / 87$ & 323 & & & 0.00 & & 0.00 \\
\hline $11 / 20 / 87$ & 324 & & & & & \\
\hline $11 / 21 / 87$ & 325 & & & & & \\
\hline $11 / 22 / 87$ & 326 & & & & & \\
\hline $11 / 23 / 87$ & 327 & & 0.00 & 0.00 & & \\
\hline $11 / 24 / 87$ & 328 & & & & & \\
\hline $11 / 25 / 87$ & 329 & & & & & \\
\hline $11 / 26 / 87$ & 330 & & & & & \\
\hline
\end{tabular}


Table 3... Precipitation for Wililams Lake gage and surrounding National Weather Service gages, $1987-88$--Continued

\begin{tabular}{|c|c|c|c|c|c|c|}
\hline Date & $\begin{array}{c}\text { Jullan } \\
\text { day }\end{array}$ & $\begin{array}{l}\text { Preclpi- } \\
\text { tatlon at } \\
\text { Williams } \\
\text { Lake gauge }\end{array}$ & $\begin{array}{l}\text { NWS } \\
\text { preclpita- } \\
\text { tion at Deep } \\
\text { Portage }\end{array}$ & $\begin{array}{l}\text { NWS } \\
\text { preclpita- } \\
\text { tlon at Park } \\
\text { Rapids }\end{array}$ & $\begin{array}{l}\text { NWS } \\
\text { preciplta- } \\
\text { tlon at } \\
\text { Pine River }\end{array}$ & $\begin{array}{l}\text { NWS } \\
\text { Preclpl- } \\
\text { tatlon at } \\
\text { Walker }\end{array}$ \\
\hline $11 / 27 / 87$ & 331 & & & & & \\
\hline $11 / 28 / 87$ & 332 & & & & & \\
\hline $11 / 29 / 87$ & 333 & & & 0.00 & & \\
\hline $11 / 30 / 87$ & 334 & & 0.00 & 0.00 & & 0.00 \\
\hline $12 / 1 / 87$ & 335 & & , & & & \\
\hline $12 / 2 / 87$ & 336 & & & 0.04 & & \\
\hline $12 / 3 / 87$ & 337 & & & 0.13 & & \\
\hline $12 / 4 / 87$ & 338 & & & & & \\
\hline $12 / 5 / 87$ & 339 & & & & & \\
\hline $12 / 6 / 87$ & 340 & & 0.84 & 1.43 & & 0.70 \\
\hline $12 / 7 / 87$ & 341 & & & 0.00 & & \\
\hline $12 / 8 / 87$ & 342 & & 0.08 & 0.00 & & 0.01 \\
\hline $12 / 9 / 87$ & 343 & & 0.00 & 0.00 & & 0.01 \\
\hline $12 / 10 / 87$ & 344 & & & & & \\
\hline $12 / 11 / 87$ & 345 & & & 0.07 & & 0.01 \\
\hline $12 / 12 / 87$ & 346 & & 0.05 & 0.03 & & 0.02 \\
\hline $12 / 13 / 87$ & 347 & & 0.04 & 0.01 & & \\
\hline $12 / 14 / 87$ & 348 & & 0.05 & 0.00 & & 0.00 \\
\hline $12 / 15 / 87$ & 349 & & 0.04 & 0.00 & & 0.00 \\
\hline $12 / 16 / 87$ & 350 & & 0.03 & 0.00 & & \\
\hline $12 / 17 / 87$ & 351 & & 0.02 & 0.00 & & \\
\hline $12 / 18 / 87$ & 352 & & & 0.00 & & \\
\hline $12 / 19 / 87$ & 353 & & & & & \\
\hline $12 / 20 / 87$ & 354 & & 0.01 & 0.00 & & \\
\hline $12 / 21 / 87$ & 355 & & 0.05 & & & \\
\hline $12 / 22 / 87$ & 356 & & & & & 0.00 \\
\hline $12 / 23 / 87$ & 357 & & & & & \\
\hline $12 / 24 / 87$ & 358 & & & & & \\
\hline $12 / 25 / 87$ & 359 & & & & & \\
\hline $12 / 26 / 87$ & 360 & & & & & \\
\hline $12 / 27 / 87$ & 361 & & & & & \\
\hline $12 / 28 / 87$ & 362 & & 0.00 & & & \\
\hline $12 / 29 / 87$ & 363 & & & & & \\
\hline $12 / 30 / 87$ & 364 & & & 0.00 & & 0.03 \\
\hline $12 / 31 / 87$ & 365 & & & 0.19 & & 0.10 \\
\hline $1 / 1 / 88$ & 1 & & & 0.03 & & \\
\hline $1 / 2 / 88$ & 2 & & & & & \\
\hline $1 / 3 / 88$ & 3 & & & & & \\
\hline $1 / 4 / 88$ & 4 & & & & & \\
\hline $1 / 5 / 88$ & 5 & & & & & \\
\hline $1 / 6 / 88$ & 6 & & & & & \\
\hline $1 / 7 / 88$ & 7 & & & & & \\
\hline $1 / 8 / 88$ & 8 & & & & & \\
\hline 1/9/88 & 9 & & & & & \\
\hline $1 / 10 / 88$ & 10 & & & & & \\
\hline $1 / 11 / 88$ & 11 & & & 0.14 & & \\
\hline $1 / 12 / 88$ & 12 & & & 0.61 & 0.50 & \\
\hline $1 / 13 / 88$ & 13 & & & & 0.11 & \\
\hline $1 / 14 / 88$ & 14 & & & & 0.02 & \\
\hline $1 / 15 / 88$ & 15 & & & & & \\
\hline $1 / 16 / 88$ & 16 & & & & & \\
\hline $1 / 17 / 88$ & 17 & & & & & \\
\hline $1 / 18 / 88$ & 18 & & & & & \\
\hline $1 / 19 / 88$ & 19 & & & & 0.11 & \\
\hline $1 / 20 / 88$ & 20 & & & & 0.27 & \\
\hline
\end{tabular}


Table 3.-. Precipitation for Williams Lake gage and surrounding National Weather

Service gages, 1987-88 -.-Continued

\begin{tabular}{|c|c|c|c|c|c|c|}
\hline Date & $\begin{array}{c}\text { Julian } \\
\text { day }\end{array}$ & $\begin{array}{l}\text { Preclpl- } \\
\text { tation at } \\
\text { Williams } \\
\text { Lake gauge }\end{array}$ & $\begin{array}{l}\text { NWS } \\
\text { precipita- } \\
\text { tlon at Deep } \\
\text { Portage }\end{array}$ & $\begin{array}{l}\text { NWS } \\
\text { preclpita- } \\
\text { tlon at Park } \\
\text { Rapids }\end{array}$ & $\begin{array}{l}\text { NWS } \\
\text { precipita- } \\
\text { tion at } \\
\text { Pine Rlver }\end{array}$ & $\begin{array}{l}\text { NWS } \\
\text { Precipl- } \\
\text { tation at } \\
\text { Walker }\end{array}$ \\
\hline $1 / 21 / 88$ & 21 & & & & & \\
\hline $1 / 22 / 88$ & 22 & & & & 0.02 & \\
\hline 1/23/88 & 23 & & & 0.07 & & 0.05 \\
\hline $1 / 24 / 88$ & 24 & & & 0.06 & 0.08 & 0.30 \\
\hline $1 / 25 / 88$ & 25 & & & & 0.09 & 0.02 \\
\hline $1 / 26 / 88$ & 26 & & & & & \\
\hline $1 / 27 / 88$ & 27 & & & & & \\
\hline $1 / 28 / 88$ & 28 & & & & & \\
\hline $1 / 29 / 88$ & 29 & & & & & \\
\hline $1 / 30 / 88$ & 30 & & & 0.11 & & 0.05 \\
\hline $1 / 31 / 88$ & 31 & & & 0.03 & 0.02 & 0.23 \\
\hline $2 / 1 / 88$ & 32 & & & & & \\
\hline $2 / 2 / 88$ & 33 & & & & & \\
\hline $2 / 3 / 88$ & 34 & & & & & \\
\hline $2 / 4 / 88$ & 35 & & & & & \\
\hline $2 / 5 / 88$ & 36 & & & & & \\
\hline $2 / 6 / 88$ & 37 & & & & & \\
\hline 2/7/88 & 38 & & 0.02 & & 0.03 & 0.03 \\
\hline $2 / 8 / 88$ & 39 & & & 0.04 & & \\
\hline 2/9/88 & 40 & & & & & \\
\hline $2 / 10 / 88$ & 41 & & & & & \\
\hline $2 / 11 / 88$ & 42 & & & & & \\
\hline $2 / 12 / 88$ & 43 & & & 0.01 & & \\
\hline $2 / 13 / 88$ & 44 & & & & & \\
\hline $2 / 14 / 88$ & 45 & & & & & \\
\hline $2 / 15 / 88$ & 46 & & & & & \\
\hline $2 / 16 / 88$ & 47 & & & & & \\
\hline 2/17/88 & 48. & & & & & \\
\hline 2/18/88 & 49 & & & & & \\
\hline 2/19/88 & 50 & & & 0.10 & & \\
\hline $2 / 20 / 88$ & 51 & & & & & \\
\hline $2 / 21 / 88$ & 52 & & & & & \\
\hline $2 / 22 / 88$ & 53 & & & & & \\
\hline 2/23/88 & 54 & & & & & \\
\hline $2 / 24 / 88$ & 55 & & & & & \\
\hline $2 / 25 / 88$ & 56 & & & & & \\
\hline 2/26/88 & 57 & & & & & \\
\hline 2/27/88 & 58 & & & & & \\
\hline $2 / 28 / 88$ & 59 & & & & & \\
\hline $2 / 29 / 88$ & 60 & & & & & \\
\hline $3 / 1 / 88$ & 61 & & & & & \\
\hline $3 / 2 / 88$ & 62 & & & & & \\
\hline $3 / 3 / 88$ & 63 & & & & & \\
\hline $3 / 4 / 88$ & 64 & & & & & \\
\hline $3 / 5 / 88$ & 65 & & & & & \\
\hline $3 / 6 / 88$ & 66 & & & & & \\
\hline $3 / 7 / 88$ & 67 & & & & & \\
\hline $3 / 8 / 88$ & 68 & & & 0.48 & 0.51 & 0.80 \\
\hline 3/9/88 & 69 & & & & 0.69 & \\
\hline $3 / 10 / 88$ & 70 & & & & & \\
\hline $3 / 11 / 88$ & 71 & & & & & 0.90 \\
\hline $3 / 12 / 88$ & 72 & & & 0.10 & 0.10 & 0.39 \\
\hline $3 / 13 / 88$ & 73 & & & & 0.33 & \\
\hline $3 / 14 / 88$ & 74 & & & & & \\
\hline $3 / 15 / 88$ & 75 & & & & & \\
\hline
\end{tabular}


Table 3.--Precipitation for Williams Lake gage and surrounding Natlonal Weather Service gages, 1987-88 --Continued

\begin{tabular}{|c|c|c|c|c|c|c|}
\hline Date & $\begin{array}{c}\text { Julian } \\
\text { day }\end{array}$ & $\begin{array}{l}\text { Preclpl. } \\
\text { tation at } \\
\text { Wllliams } \\
\text { Lake gauge }\end{array}$ & $\begin{array}{l}\text { NWS } \\
\text { precipita- } \\
\text { tlon at Deep } \\
\text { Portage }\end{array}$ & $\begin{array}{l}\text { NWS } \\
\text { preclpita- } \\
\text { tlon at Park } \\
\text { Rapida }\end{array}$ & $\begin{array}{l}\text { NWS } \\
\text { precipita- } \\
\text { tlon at } \\
\text { Pine River } \\
\end{array}$ & $\begin{array}{l}\text { NWS } \\
\text { Preclpi- } \\
\text { tation at } \\
\text { Walker }\end{array}$ \\
\hline $3 / 16 / 88$ & 76 & & & & & \\
\hline $3 / 17 / 88$ & 77 & & & & & \\
\hline $3 / 18 / 88$ & 78 & & & & 0.02 & \\
\hline 3/19/88 & 79 & & & & & \\
\hline $3 / 20 / 88$ & 80 & & & & 0.02 & \\
\hline $3 / 21 / 88$ & 81 & & & & & \\
\hline $3 / 22 / 88$ & 82 & & & & & \\
\hline 3/23/88 & 83 & & & & & \\
\hline $3 / 24 / 88$ & 84 & & & 0.50 & 0.06 & 0.82 \\
\hline $3 / 25 / 88$ & 85 & & & 0.03 & 0.92 & 0.01 \\
\hline $3 / 26 / 88$ & 86 & & & & & \\
\hline $3 / 27 / 88$ & 87 & & & & & \\
\hline $3 / 28 / 88$ & 88 & & & 0.09 & & 0.14 \\
\hline $3 / 29 / 88$ & 89 & & & & 0.13 & \\
\hline $3 / 30 / 88$ & 90 & & & & & \\
\hline $3 / 31 / 88$ & 91 & & & & & \\
\hline 4/1/88 & 92 & & & & & \\
\hline $4 / 2 / 88$ & 93 & & & 0.03 & & 0.09 \\
\hline $4 / 3 / 88$ & 94 & & 0.40 & 0.17 & 0.25 & 0.16 \\
\hline $4 / 4 / 88$ & 95 & & & & & \\
\hline $4 / 5 / 88$ & 96 & & & & & \\
\hline $4 / 6 / 88$ & 97 & & & & & \\
\hline $4 / 7 / 88$ & 98 & & & & & \\
\hline $4 / 8 / 88$ & 99 & & & & & \\
\hline 4/9/88 & 100 & & & & 0.09 & 0.12 \\
\hline 4/10/88 & 101 & & & & & \\
\hline $4 / 11 / 88$ & 102 & & & & . & \\
\hline $4 / 12 / 88$ & 103 & & & & & \\
\hline $4 / 13 / 88$ & 104 & & & & & \\
\hline $4 / 14 / 88$ & 105 & & & & & \\
\hline $4 / 15 / 88$ & 106 & & & & & \\
\hline $4 / 16 / 88$ & 107 & & & & & \\
\hline $4 / 17 / 88$ & 108 & & & & & \\
\hline $4 / 18 / 88$ & 109 & & & & & \\
\hline $4 / 19 / 88$ & 110 & & & & & \\
\hline $4 / 20 / 88$ & 111 & & & & & \\
\hline $4 / 21 / 88$ & 112 & & & & & \\
\hline $4 / 22 / 88$ & 113 & & & & & \\
\hline $4 / 23 / 88$ & 114 & & & & & \\
\hline $4 / 24 / 88$ & 115 & & & & & \\
\hline $4 / 25 / 88$ & 116 & & & & & 0.02 \\
\hline $4 / 26 / 88$ & 117 & & & & & \\
\hline $4 / 27 / 88$ & 118 & & & & & \\
\hline 4/28/88 & 119 & & & & & \\
\hline 4/29/88 & 120 & & & & & \\
\hline $4 / 30 / 88$ & 121 & & & & & \\
\hline $5 / 1 / 88$ & 122 & & & & & \\
\hline $5 / 2 / 88$ & 123 & & & & & \\
\hline $5 / 3 / 88$ & 124 & & & & & \\
\hline $5 / 4 / 88$ & 125 & & & & & \\
\hline $5 / 5 / 88$ & 126 & & & & & \\
\hline $5 / 6 / 88$ & 127 & & & & & \\
\hline $5 / 7 / 88$ & 128 & 0.36 & 0.01 & & 0.05 & 0.48 \\
\hline $5 / 8 / 88$ & 129 & 0.06 & 0.08 & 0.10 & 0.29 & \\
\hline $5 / 9 / 88$ & 130 & 0.03 & 0.02 & 0.18 & 0.57 & 0.03 \\
\hline
\end{tabular}


Table 3.-. Preclpltatlon for Williams Lake gage and surrounding Natlonal Weather Service gages, 1987.88 -.Continued

\begin{tabular}{|c|c|c|c|c|c|c|}
\hline Date & $\begin{array}{c}\text { Julian } \\
\text { day }\end{array}$ & $\begin{array}{l}\text { Precipl- } \\
\text { tation at } \\
\text { Williams } \\
\text { Lake gauge }\end{array}$ & $\begin{array}{l}\text { NWS } \\
\text { preclpita- } \\
\text { tlon at Deep } \\
\text { Portage }\end{array}$ & $\begin{array}{l}\text { NWS } \\
\text { preclplta- } \\
\text { tlon at Park } \\
\text { Rapids }\end{array}$ & $\begin{array}{l}\text { NWS } \\
\text { precipita- } \\
\text { tlon at } \\
\text { Pine River }\end{array}$ & $\begin{array}{l}\text { NWS } \\
\text { Precipl- } \\
\text { tation at } \\
\text { Walker }\end{array}$ \\
\hline $5 / 10 / 88$ & 131 & & & & 0.02 & \\
\hline $5 / 11 / 88$ & 132 & & & & & \\
\hline $5 / 12 / 88$ & 133 & 0.22 & 0.11 & 1.20 & 0.10 & 0.28 \\
\hline $5 / 13 / 88$ & 134 & 0.07 & 0.17 & & 0.23 & \\
\hline $5 / 14 / 88$ & 135 & 0.15 & & 0.03 & & 0.02 \\
\hline $5 / 15 / 88$ & 136 & 0.29 & · & 0.04 & & 0.03 \\
\hline $5 / 16 / 88$ & 137 & & & & & \\
\hline $5 / 17 / 88$ & 138 & & 0.01 & & & \\
\hline $5 / 18 / 88$ & 139 & & & & & \\
\hline $5 / 19 / 88$ & 140 & & 0.01 & & 0.07 & \\
\hline $5 / 20 / 88$ & 141 & & 0.01 & 0.33 & & \\
\hline $5 / 21 / 88$ & 142 & & 0.01 & 0.12 & 0.03 & 0.11 \\
\hline $5 / 22 / 88$ & 143 & 0.02 & & 0.28 & & 0.26 \\
\hline $5 / 23 / 88$ & 144 & & & & & \\
\hline $5 / 24 / 88$ & 145 & & & & & \\
\hline $5 / 25 / 88$ & 146 & & & & & \\
\hline $5 / 26 / 88$ & 147 & & & & & \\
\hline $5 / 27 / 88$ & 148 & 0.02 & 0.15 & 0.38 & 1.11 & 0.13 \\
\hline $5 / 28 / 88$ & 149 & & & & & \\
\hline $5 / 29 / 88$ & 150 & & & & & \\
\hline $5 / 30 / 88$ & 151 & & & & & \\
\hline $5 / 31 / 88$ & 152 & & & & & \\
\hline $6 / 1 / 88$ & 153 & & & & & \\
\hline $6 / 2 / 88$ & 154 & & & 0.24 & & \\
\hline $6 / 3 / 88$ & 155 & & & & & \\
\hline $6 / 4 / 88$ & 156 & & & & & \\
\hline $6 / 5 / 88$ & 157 & & & & & \\
\hline $6 / 6 / 88$ & 158 & & & & & \\
\hline $6 / 7 / 88$ & 159 & & & & & \\
\hline $6 / 8 / 88$ & 160 & 0.02 & & & & 0.07 \\
\hline $6 / 9 / 88$ & 161 & & 0.19 & & 0.15 & \\
\hline $6 / 10 / 88$ & 162 & & & & & \\
\hline $6 / 11 / 88$ & 163 & & & & & \\
\hline $6 / 12 / 88$ & 164 & & & & & \\
\hline $6 / 13 / 88$ & 165 & 0.11 & & & 0.09 & 0.04 \\
\hline $6 / 14 / 88$ & 166 & 0.26 & 0.36 & 0.24 & 0.49 & 0.35 \\
\hline $6 / 15 / 88$ & 167 & 0.07 & 0.04 & 0.08 & & 0.04 \\
\hline $6 / 16 / 88$ & 168 & & 0.09 & & 0.14 & 0.01 \\
\hline $6 / 17 / 88$ & 169 & & & & & \\
\hline $6 / 18 / 88$ & 170 & 0.56 & 0.05 & & & \\
\hline $6 / 19 / 88$ & 171 & & 0.54 & 0.06 & 0.52 & 0.62 \\
\hline $6 / 20 / 88$ & 172 & & & & & \\
\hline $6 / 21 / 88$ & 173 & 1.32 & 0.56 & 1.16 & 0.36 & 0.54 \\
\hline $6 / 22 / 88$ & 174 & & 0.25 & 0.02 & 0.25 & \\
\hline $6 / 23 / 88$ & 175 & & 0.04 & & & 0.01 \\
\hline $6 / 24 / 88$ & 176 & & 0.03 & & & \\
\hline $6 / 25 / 88$ & 177 & & & & & \\
\hline $6 / 26 / 88$ & 178 & & & & & \\
\hline $6 / 27 / 88$ & 179 & & & & & \\
\hline $6 / 28 / 88$ & 180 & 0.12 & 0.13 & & 0.37 & 0.08 \\
\hline $6 / 29 / 88$ & 181 & & 0.03 & 0.13 & & \\
\hline $6 / 30 / 88$ & 182 & & & & & \\
\hline $7 / 1 / 88$ & 183 & & & & & \\
\hline $7 / 2 / 88$ & 184 & & & & & \\
\hline $7 / 3 / 88$ & 185 & & & & & \\
\hline
\end{tabular}


Table 3...Precipitation for Williams Lake gage and surrounding National Weather Service gages, 1987.88 --Continued

\begin{tabular}{|c|c|c|c|c|c|c|}
\hline Date & $\begin{array}{c}\text { Jullan } \\
\text { day }\end{array}$ & $\begin{array}{l}\text { Preclpl- } \\
\text { tation at } \\
\text { Williams } \\
\text { Lake gauge }\end{array}$ & $\begin{array}{l}\text { NWS } \\
\text { preclpita- } \\
\text { fion at Deep } \\
\text { Portage }\end{array}$ & $\begin{array}{l}\text { NWS } \\
\text { precipita- } \\
\text { tion at Park } \\
\text { Rapids }\end{array}$ & $\begin{array}{l}\text { NWS } \\
\text { precipita- } \\
\text { tion at } \\
\text { Pine River }\end{array}$ & $\begin{array}{l}\text { NWS } \\
\text { Precipl- } \\
\text { tation at } \\
\text { Walker }\end{array}$ \\
\hline $7 / 4 / 88$ & 186 & & & & & \\
\hline $7 / 5 / 88$ & 187 & & & & & \\
\hline $7 / 6 / 88$ & 188 & 0.40 & & & & 1.47 \\
\hline $7 / 7 / 88$ & 189 & & 0.02 & 1.73 & & \\
\hline $7 / 8 / 88$ & 190 & & & & & \\
\hline $7 / 9 / 88$ & 191 & & ' & & & \\
\hline $7 / 10 / 88$ & 192 & & & & 0.40 & \\
\hline $7 / 11 / 88$ & 193 & & & & & \\
\hline $7 / 12 / 88$ & 194 & 0.47 & & & & \\
\hline $7 / 13 / 88$ & 195 & 0.07 & 0.79 & 0.54 & 0.82 & 0.35 \\
\hline $7 / 14 / 88$ & 196 & & 0.07 & & & \\
\hline $7 / 15 / 88$ & 197 & & & & & \\
\hline $7 / 16 / 88$ & 198 & & & & & \\
\hline $7 / 17 / 88$ & 199 & & & & & \\
\hline $7 / 18 / 88$ & 200 & & & & & \\
\hline $7 / 19 / 88$ & 201 & 0.05 & & 0.08 & & 0.03 \\
\hline $7 / 20 / 88$ & 202 & & 0.09 & 0.28 & 0.10 & 0.29 \\
\hline $7 / 21 / 88$ & 203 & & & & & \\
\hline $7 / 22 / 88$ & 204 & 0.01 & 0.06 & & 0.16 & \\
\hline $7 / 23 / 88$ & 205 & 0.39 & & & & \\
\hline $7 / 24 / 88$ & 206 & & 0.29 & 0.15 & 0.09 & 0.93 \\
\hline $7 / 25 / 88$ & 207 & & & & & \\
\hline $7 / 26 / 88$ & 208 & & & & & \\
\hline $7 / 27 / 88$ & 209 & & & & & \\
\hline $7 / 28 / 88$ & 210 & 0.05 & 0.04 & & & \\
\hline $7 / 29 / 88$ & 211 & 0.02 & & & 0.09 & 0.40 \\
\hline $7 / 30 / 88$ & 212 & & & & & \\
\hline $7 / 31 / 88$ & 213 & 0.36 & 0.39 & 0.26 & 0.55 & 0.52 \\
\hline $8 / 1 / 88$ & 214 & 1.54 & 0.02 & 0.36 & 0.02 & 0.10 \\
\hline $8 / 2 / 88$ & 215 & 0.44 & 0.78 & 2.69 & 0.40 & 1.76 \\
\hline $8 / 3 / 88$ & 216 & 0.11 & 0.08 & 0.04 & 0.15 & \\
\hline $8 / 4 / 88$ & 217 & & 0.88 & 0.08 & 0.28 & 0.29 \\
\hline $8 / 5 / 88$ & 218 & & 0.24 & 0.43 & 0.36 & 0.80 \\
\hline $8 / 6 / 88$ & 219 & & 0.05 & & & \\
\hline $8 / 7 / 88$ & 220 & & & 0.85 & & 1.40 \\
\hline $8 / 8 / 88$ & 221 & & 0.70 & & 0.38 & \\
\hline $8 / 9 / 88$ & 222 & & & & 0.01 & \\
\hline $8 / 10 / 88$ & 223 & & & & & \\
\hline $8 / 11 / 88$ & 224 & 0.25 & 0.07 & 0.10 & & \\
\hline $8 / 12 / 88$ & 225 & 0.04 & 0.38 & 0.19 & 1.35 & 0.53 \\
\hline $8 / 13 / 88$ & 226 & 2.17 & 0.42 & 1.54 & & 2.76 \\
\hline $8 / 14 / 88$ & 227 & 0.01 & 1.32 & & 1.98 & 0.01 \\
\hline $8 / 15 / 88$ & 228 & & & & & \\
\hline $8 / 16 / 88$ & 229 & & & & & \\
\hline $8 / 17 / 88$ & 230 & 0.02 & & 0.03 & & 0.01 \\
\hline $8 / 18 / 88$ & 231 & & & & & 0.01 \\
\hline $8 / 19 / 88$ & 232 & & & & & \\
\hline $8 / 20 / 88$ & 233 & & & & & \\
\hline $8 / 21 / 88$ & 234 & 0.11 & & 0.15 & & 0.03 \\
\hline $8 / 22 / 88$ & 235 & 0.17 & 0.08 & 0.39 & 0.08 & 0.20 \\
\hline $8 / 23 / 88$ & 236 & & 0.57 & 0.16 & 0.79 & 0.06 \\
\hline $8 / 24 / 88$ & 237 & & & & & \\
\hline $8 / 25 / 88$ & 238 & & & & & \\
\hline $8 / 26 / 88$ & 239 & & & & & 0.02 \\
\hline $8 / 27 / 88$ & 240 & & & & 0.01 & \\
\hline
\end{tabular}


Table 3... Precipitation for Williams Lake gage and surrounding National Weather Service gages, 1987.88 -.Continued

\begin{tabular}{|c|c|c|c|c|c|c|}
\hline Date & $\begin{array}{c}\text { Jullan } \\
\text { day }\end{array}$ & $\begin{array}{l}\text { Preclpl- } \\
\text { tation at } \\
\text { Williams } \\
\text { Lake gauge }\end{array}$ & $\begin{array}{l}\text { NWS } \\
\text { precipita- } \\
\text { ton at Deep } \\
\text { Portage }\end{array}$ & $\begin{array}{l}\text { NWS } \\
\text { preciplta- } \\
\text { tlon at Park } \\
\text { Rapids }\end{array}$ & $\begin{array}{l}\text { NWS } \\
\text { precipita- } \\
\text { tion at } \\
\text { Pine River }\end{array}$ & $\begin{array}{l}\text { NWS } \\
\text { Precipl- } \\
\text { tation at } \\
\text { Waiker }\end{array}$ \\
\hline $8 / 28 / 88$ & 241 & & & & & \\
\hline $8 / 29 / 88$ & 242 & & & & & \\
\hline $8 / 30 / 88$ & 243 & & & & & \\
\hline $8 / 31 / 88$ & 244 & & & & & \\
\hline $9 / 1 / 88$ & 245 & 0.70 & 0.72 & & 0.47 & 0.75 \\
\hline $9 / 2 / 88$ & 246 & 0.06 & $\cdot$ & & & 0.03 \\
\hline $9 / 3 / 88$ & 247 & 0.16 & 0.15 & & 0.27 & 0.14 \\
\hline $9 / 4 / 88$ & 248 & 0.31 & & & & \\
\hline $9 / 5 / 88$ & 249 & 0.11 & & & & \\
\hline $9 / 6 / 88$ & 250 & & & & & \\
\hline $9 / 7 / 88$ & 251 & 1.21 & & & & \\
\hline $9 / 8 / 88$ & 252 & & & & & \\
\hline $9 / 9 / 88$ & 253 & & & & & \\
\hline $9 / 10 / 88$ & 254 & & & & & \\
\hline $9 / 11 / 88$ & 255 & & & & & \\
\hline $9 / 12 / 88$ & 256 & & & & 0.09 & 0.08 \\
\hline $9 / 13 / 88$ & 257 & & & & & \\
\hline $9 / 14 / 88$ & 258 & & & & & \\
\hline $9 / 15 / 88$ & 259 & 0.24 & & & & 0.03 \\
\hline $9 / 16 / 88$ & 260 & 0.44 & 0.56 & & 0.65 & 0.61 \\
\hline $9 / 17 / 88$ & 261 & 0.19 & & & 0.64 & 0.14 \\
\hline $9 / 18 / 88$ & 262 & 0.05 & 0.89 & & & 0.18 \\
\hline $9 / 19 / 88$ & 263 & 0.98 & & & 1.11 & 0.68 \\
\hline $9 / 20 / 88$ & 264 & 0.09 & 0.53 & & 1.14 & \\
\hline $9 / 21 / 88$ & 265 & & & & & 0.03 \\
\hline $9 / 22 / 88$ & 266 & & 0.06 & & 0.02 & 0.11 \\
\hline $9 / 23 / 88$ & 267 & & & & & \\
\hline $9 / 24 / 88$ & 268 & & & & & \\
\hline $9 / 25 / 88$ & 269 & & & & & 0.01 \\
\hline $9 / 26 / 88$ & 270 & & & & & \\
\hline $9 / 27 / 88$ & 271 & & & & & \\
\hline $9 / 28 / 88$ & 272 & 0.50 & & & & 0.06 \\
\hline $9 / 29 / 88$ & 273 & 0.01 & 0.51 & & 0.89 & 0.37 \\
\hline $9 / 30 / 88$ & 274 & & & & 0.02 & \\
\hline $10 / 1 / 88$ & 275 & & & & & \\
\hline $10 / 2 / 88$ & 276 & & & & & \\
\hline $10 / 3 / 88$ & 277 & & & & & \\
\hline $10 / 4 / 88$ & 278 & & & & & \\
\hline $10 / 5 / 88$ & 279 & & & & & \\
\hline $10 / 6 / 88$ & 280 & & & & & \\
\hline $10 / 7 / 88$ & 281 & & & & & \\
\hline $10 / 8 / 88$ & 282 & & & & & \\
\hline $10 / 9 / 88$ & 283 & & & & & \\
\hline $10 / 10 / 88$ & 284 & & & & & \\
\hline $10 / 11 / 88$ & 285 & & & & & \\
\hline $10 / 12 / 88$ & 286 & & 0.08 & & & \\
\hline $10 / 13 / 88$ & 287 & & & & & \\
\hline $10 / 14 / 88$ & 288 & & & & & \\
\hline $10 / 15 / 88$ & 289 & & & & & \\
\hline $10 / 16 / 88$ & 290 & 0.01 & & & 0.05 & 0.12 \\
\hline $10 / 17 / 88$ & 291 & 0.13 & & 0.18 & 0.04 & 0.06 \\
\hline $10 / 18 / 88$ & 292 & 0.02 & 0.14 & 0.06 & 0.19 & 0.05 \\
\hline $10 / 19 / 88$ & 293 & & & & 0.20 & \\
\hline $10 / 20 / 88$ & 294 & 0.34 & 0.40 & 0.32 & & 0.30 \\
\hline $10 / 21 / 88$ & 295 & 0.01 & & & 0.44 & \\
\hline
\end{tabular}


Table 3.--Precipitation for Williams Lake gage and surrounding National Weather Service gages, 1987-88 --Continued

\begin{tabular}{|c|c|c|c|c|c|c|}
\hline Date & $\begin{array}{c}\text { Jullan } \\
\text { day }\end{array}$ & $\begin{array}{l}\text { Preclpl- } \\
\text { tation at } \\
\text { Williams } \\
\text { Lake gauge }\end{array}$ & $\begin{array}{l}\text { NWS } \\
\text { precipita- } \\
\text { tion at Deep } \\
\text { Portage }\end{array}$ & $\begin{array}{l}\text { NWS } \\
\text { preclpita- } \\
\text { tlon at Park } \\
\text { Raplds }\end{array}$ & $\begin{array}{l}\text { NWS } \\
\text { precipita- } \\
\text { tion at } \\
\text { PIne River }\end{array}$ & $\begin{array}{l}\text { NWS } \\
\text { Precipl- } \\
\text { tation at } \\
\text { Walker }\end{array}$ \\
\hline $10 / 22 / 88$ & 296 & 0.04 & & & & \\
\hline $10 / 23 / 88$ & 297 & & 0.09 & & 0.07 & 0.03 \\
\hline $10 / 24 / 88$ & 298 & & & & & \\
\hline $10 / 25 / 88$ & 299 & & & & & \\
\hline $10 / 26 / 88$ & 300 & & & & & \\
\hline $10 / 27 / 88$ & 301 & 0.06 & & 0.12 & & 0.06 \\
\hline $10 / 28 / 88$ & 302 & & & 0.09 & & \\
\hline $10 / 29 / 88$ & 303 & & & & & \\
\hline $10 / 30 / 88$ & 304 & & & & & \\
\hline $10 / 31 / 88$ & 305 & $\cdot$ & 0.30 & & & \\
\hline $11 / 1 / 88$ & 306 & & & & & \\
\hline $11 / 2 / 88$ & 307 & & & & & \\
\hline $11 / 3 / 88$ & 308 & & & & & \\
\hline $11 / 4 / 88$ & 309 & & & & & \\
\hline $11 / 5 / 88$ & 310 & & & 0.02 & & \\
\hline $11 / 6 / 88$ & 311 & & & & & \\
\hline $11 / 7 / 88$ & 312 & & & & & 0.01 \\
\hline $11 / 8 / 88$ & 313 & & & 0.05 & 0.05 & \\
\hline $11 / 9 / 88$ & 314 & & & & 0.18 & 0.11 \\
\hline $11 / 10 / 88$ & 315 & & & 0.07 & & \\
\hline $11 / 11 / 88$ & 316 & & & & & \\
\hline $11 / 12 / 88$ & 317 & & 0.20 & & 0.80 & 0.47 \\
\hline $11 / 13 / 88$ & 318 & & 0.30 & 0.50 & 0.11 & \\
\hline $11 / 14 / 88$ & 319 & & & & & \\
\hline $11 / 15 / 88$ & 320 & & & & & 0.16 \\
\hline $11 / 16 / 88$ & 321 & & & 0.16 & 0.60 & \\
\hline $11 / 17 / 88$ & 322 & & & 0.12 & 0.04 & \\
\hline $11 / 18 / 88$ & 323 & & & & & \\
\hline $11 / 19 / 88$ & 324 & & 0.25 & 0.07 & 0.44 & \\
\hline $11 / 20 / 88$ & 325 & & & 0.12 & , & \\
\hline $11 / 21 / 88$ & 326 & & & & & \\
\hline $11 / 22 / 88$ & 327 & & & & 0.13 & \\
\hline $11 / 23 / 88$ & 328 & & & & & \\
\hline $11 / 24 / 88$ & 329 & . & & & & \\
\hline $11 / 25 / 88$ & 330 & & & & & \\
\hline $11 / 26 / 88$ & 331 & & & 0.20 & 0.25 & \\
\hline $11 / 27 / 88$ & 332 & & $\cdot$ & 0.30 & 2.75 & 0.30 \\
\hline $11 / 28 / 88$ & 333 & & 0.72 & & 0.30 & 0.20 \\
\hline $11 / 29 / 88$ & 334 & & & & & \\
\hline $11 / 30 / 88$ & 335 & & & 0.01 & & \\
\hline $12 / 1 / 88$ & 336 & & & & & \\
\hline $12 / 2 / 88$ & 337 & & & & & \\
\hline $12 / 3 / 88$ & 338 & & & & & \\
\hline $12 / 4 / 88$ & 339 & & & & & \\
\hline $12 / 5 / 88$ & 340 & & & & & 0.01 \\
\hline $12 / 6 / 88$ & 341 & & & & & \\
\hline $12 / 7 / 88$ & 342 & & & & & 0.01 \\
\hline $12 / 8 / 88$ & 343 & & & & & 0.01 \\
\hline $12 / 9 / 88$ & 344 & & & & & \\
\hline $12 / 10 / 88$ & 345 & & & & & \\
\hline $12 / 11 / 88$ & 346 & & & & & \\
\hline $12 / 12 / 88$ & 347 & & & 0.09 & & \\
\hline $12 / 13 / 88$ & 348 & & & & 0.03 & \\
\hline $12 / 14 / 88$ & 349 & & 0.48 & 0.29 & 0.42 & 0.37 \\
\hline $12 / 15 / 88$ & 350 & & & & & \\
\hline
\end{tabular}


Table 3.--Precipitation for Wiiilams Lake gage and surrounding National Weather Service gages, 1987-88 -.Continued

\begin{tabular}{|c|c|c|c|c|c|c|}
\hline Date & $\begin{array}{c}\text { Jullan } \\
\text { day }\end{array}$ & $\begin{array}{l}\text { Preclpi. } \\
\text { tation at } \\
\text { Williams } \\
\text { Lake gauge }\end{array}$ & $\begin{array}{l}\text { NWS } \\
\text { preciplta- } \\
\text { tion at Deep } \\
\text { Portage }\end{array}$ & $\begin{array}{l}\text { NWS } \\
\text { preciplia- } \\
\text { tion at Park } \\
\text { Rapids }\end{array}$ & $\begin{array}{l}\text { NWS } \\
\text { precipita- } \\
\text { tion at } \\
\text { Pine River }\end{array}$ & $\begin{array}{l}\text { NWS } \\
\text { Precipi- } \\
\text { tation at } \\
\text { Walker }\end{array}$ \\
\hline $12 / 16 / 88$ & 351 & & 0.09 & 0.06 & 0.04 & \\
\hline $12 / 17 / 88$ & 352 & & & & & \\
\hline $12 / 18 / 88$ & 353 & & 0.05 & & 0.05 & \\
\hline $12 / 19 / 88$ & 354 & & & & & \\
\hline $12 / 20 / 88$ & 355 & & & & & \\
\hline $12 / 21 / 88$ & 356 & & 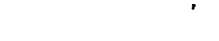 & & & \\
\hline $12 / 22 / 88$ & 357 & & & & & \\
\hline $12 / 23 / 88$ & 358 & & & & 0.19 & 0.05 \\
\hline $12 / 24 / 88$ & 359 & & & & & \\
\hline $12 / 25 / 88$ & 360 & & & & & \\
\hline $12 / 26 / 88$ & 361 & & & & 0.03 & 0.30 \\
\hline $12 / 27 / 88$ & 362 & & 0.55 & 0.32 & 0.47 & 0.25 \\
\hline $12 / 28 / 88$ & 363 & & & & & \\
\hline $12 / 29 / 88$ & 364 & & & & 0.02 & \\
\hline $12 / 30 / 88$ & 365 & & 0.02 & & & \\
\hline $12 / 31 / 88$ & 366 & & & & & \\
\hline
\end{tabular}

